\title{
ON THE RIGIDITY THEOREMS OF WITTEN
}

\author{
RAOUL BOTT AND CLIFFORD TAUBES
}

\section{INTRODUCTION}

In this paper we prove the rigidity theorems predicted by Witten in 1986, about the index of certain elliptic operators on manifolds with an $S^{1}$ action [W].

Witten's insight was the culmination of an interesting interchange of ideas between him and Hopkins, Landweber, Ochanine, and Stong. For the detailed history, we refer the reader to [La].

The present account is essentially a reinterpretation of the second author's (Taubes' [T]) original proof of the theorem. The senior author's contribution was solely to notice that the rather densely written arguments of the original manuscript could be formulated in terms of the well known fixed point formulae of equivariant index theory and equivariant colomology. In this context, the final proof then appears in direct lineage of the Atiyah-Hirzebruch theorem concerning the vanishing of the $\widehat{A}$ genus of spin manifolds admitting a circle action $[\mathrm{A}-\mathrm{H}]$ and of the even older idea of Lusztig concerning circle actions in the complex case. There remains, however, the beautiful, physics inspired novelty of connecting these techniques with elliptic function theory.

\section{STATEMENT OF THE THEOREM}

Recall that for an elliptic operator

$$
D: \Gamma(E) \rightarrow \Gamma(F)
$$

acting on sections of vector bundles $E$ and $F$ over the compact manifold $M$, the index of $D$ is defined as the virtual vector space

$$
\text { index } D=\operatorname{ker} D-\operatorname{coker} D \text {. }
$$

If $M$ admits a circle action preserving $D$, that is, acting on $E$ and $F$ and commuting with $D$, then this index clearly inherits an $S^{1}$ action and so becomes a virtual $S^{1}$-module. As such it has a Fourier decomposition into a finite sum of irreducible one-dimensional representations

$$
\text { index } D=\sum a_{n} L^{n}
$$

Received by the editors July 18,1988 .

1980 Mathematics Subject Classification (1985 Revision). Primary 55N91, 55N99, 55M20, 19K56. 
where $L^{n}$ denotes the representation of $S^{1}$ on $\mathrm{C}$ sending $e^{i \theta}$ to $e^{i n \theta}$, and the $a_{n}$ 's are integers.

We call the operator $D$ "rigid" if, in this Fourier expansion, all $a_{n}$ with $n \neq 0$ vanish. Another way of saying this is that $D$ is rigid only if every nontrivial irreducible representation of $S^{1}$ occurring in $\operatorname{ker}(D)$ also occurs in $\operatorname{coker}(D)$ with the same multiplicity.

The simplest examples of rigid operators arise in deRham theory as a consequence of the homotopy invariance of cohomology: If

$$
\Omega^{0} \stackrel{d}{\rightarrow} \Omega^{1} \stackrel{d}{\rightarrow} \ldots \stackrel{d}{\rightarrow} \Omega^{n}
$$

is the usual deRham complex and $d^{*}$ denotes the adjoint of the exterior derivative, $d$, relative to a Riemannian structure then

$$
d+d^{*}: \Omega^{\text {even }} \rightarrow \Omega^{\text {odd }} .
$$

Acting from even-dimensional forms to odd-dimensional ones, it is rigid in our sense for any action of $S^{1}$ on $M$ by isometries. This follows from the Hodge theory because the kernel and cokernel of this operator consist precisely of the harmonic forms, which, by homotopy invariance, must stay put under the $S^{1}$ action.

Similarly, if $\Omega_{C}^{ \pm}$are the complex forms on $M$ which are even and odd, respectively, under the Hodge $*$, then the signature operator

$$
d_{S}: \Omega_{\mathbf{C}}^{+} \rightarrow \Omega_{\mathbf{C}}^{-}
$$

of an oriented manifold is rigid under any $S^{1}$-action by isometries. (When an operator is rigid under any action of $S^{1}$ by isometries, we will say that it is universally rigid.)

Here again it is the homotopy invariance that forces the rigidity because $\operatorname{ker} d_{S}$ and coker $d_{S}$ are again naturally identified with the subspace of harmonic forms on $M$ which are invariant, and anti-invariant, respectively, under the Hodge $*$ operator of $M$.

Note that in both these examples, the index is rigid in the very strong sense that both the kernel and the cokernel are separately rigid. It is for this reason that the example provided by the Dirac operator on a compact spin manifold of even dimension is more interesting. This operator,

$$
\not \partial: \Gamma\left(\Delta^{+}\right) \rightarrow \Gamma\left(\Delta^{-}\right),
$$

acts between sections of the two spin bundles of the spin structure, and it is also universally rigid. Here, the kernel and cokernel can separately "move," although they must be isomorphic $S^{1}$ modules. Indeed, this assertion is a corollary of the Atiyah-Hirzebruch Theorem which states that

$$
\text { index } \not=0 \text { whenever } M \text { is connected and admits a nontrivial } S^{1} \text { action. }
$$

Now, for $R$ a representation of $O(n)$, we also write $R$, or $R(T)$, for the bundle associated to the frame bundle of a Riemann manifold $M$ by $R$. Use 
of the Levi-Civita connection allows a functorial twisting by $R$ of each of the three operators just described. Thus, for example, the twist of $d_{S}$ by $R$, denoted by $d_{S} \otimes R$, is a new elliptic operator

$$
d_{S} \otimes R: \Omega_{\mathrm{C}}^{+}(R) \rightarrow \Omega_{\mathrm{C}}^{-}(R) .
$$

The question now arises which of these twists remain universally rigid.

Witten does not quite answer this query. It is still an open question, but he predicted that for the sequence of representations $\left\{R_{n}\right\}$ characterized below, $d_{S} \otimes R_{n}$ will be rigid.

Precisely, let $T$ denote the standard complex representation of $O(n)$ or, if you wish, the tangent bundle of $M$. We write $S^{k}(T)$ and $\Lambda^{k}(T)$ for the symmetric and exterior powers of this representation and, as is usual, set

$$
\begin{aligned}
& S_{a}(T)=\sum_{k=0}^{\infty} a^{k} S^{k}(T), \\
& \Lambda_{a}(T)=\sum_{k=0}^{\infty} a^{k} \Lambda^{k}(T) .
\end{aligned}
$$

These should be thought of as formal power series in $a$ with values in the representation ring of $O(2 n)$ or Spin $(2 n)$. We also write $\Delta^{+}$and $\Delta^{-}$for the spin representations of $\operatorname{Spin}(2 n)$ and set

$$
\Delta_{1}=\Delta^{+}+\Delta^{-} \text {and } \Delta_{-1}=\Delta^{+}-\Delta^{-} \text {. }
$$

On a spin manifold of dimension $2 n$ with Dirac operator $\not \partial$ one then has the "twisting relations"

$$
d_{S}=\not \partial \otimes \Delta_{1}, \quad d+d^{*}=\not \partial \otimes \Delta_{-1}
$$

(which, in a sense, establish $\not \partial$ as the primary elliptic operator associated to a Riemann structure on $M$ ).

With all this understood we can state the theorem that Witten conjectured as follows.

Theorem. Let $M$ be a $2 n$ dimensional, compact Riemannian manifold which admits a spin-structure with corresponding Dirac operator $\not \partial$. Also let $R_{n}$ and $R_{n}^{\prime}$ be sequences of representations defined by the formal series

$$
\begin{gathered}
R_{q} \equiv \sum_{n=0}^{\infty} q^{n} R_{n}=\bigotimes_{n=1}^{\infty} \Lambda_{q^{n}}(T) \bigotimes_{m=1}^{\infty} S_{q^{m}}(T) \\
R_{q}^{\prime} \equiv \sum_{n=0}^{\infty} q^{n / 2} R_{n}^{\prime}=\bigotimes_{n=1 / 2,3 / 2, \ldots} \Lambda_{q^{n}}(T) \bigotimes_{m=1}^{\infty} S_{q^{m}}(T) .
\end{gathered}
$$

Then each of the operators

is universally rigid.

$$
\not \partial \otimes R_{n}^{\prime} \text { and } \not \partial \otimes \Delta_{1} \otimes R_{n} \equiv d_{S} \otimes R_{n}
$$


The theorem has been established under restricted hypotheses about the $S^{1}$-action by Landweber and Stong [L-S] and then by Ochanine [O].

Remarks. (1) The first few of these representations are given by

$$
\begin{aligned}
& R_{0}^{\prime}=1, \\
& R_{1}^{\prime}=T, \\
& R_{2}^{\prime}=\Lambda^{2} T \oplus T, \\
& R_{3}^{\prime}=\Lambda^{3} T \oplus T \otimes T \oplus T,
\end{aligned}
$$

and

$$
\begin{aligned}
& R_{0}=1 \\
& R_{1}=2 T \\
& R_{2}=2\left(T^{2}+T\right) \\
& R_{3}=2 T^{3}+\Lambda^{3} T+S^{3} T+4 T^{2}+2 T
\end{aligned}
$$

It is clear, therefore, that this is a complicated series of representations and one might at first think it is for this reason that their rigidity was not noted earlier in some other context. Amazingly, this is not so. For instance, even the rigidity of the first new candidate $d_{S} \otimes T$, cannot be established in isolation, so to speak, at least as far as we know. Note also that these operators are rigid only on spin manifolds, although the family $d_{S} \otimes R_{n}$ is perfectly well defined on all oriented ones.

(2) Where do these formulae come from? Certainly they make best conceptual sense from Witten's physics-inspired point of view as formulae on the loop space $\mathscr{L} M$. But, as we will see, they also make excellent sense purely in the context of the "fixed point formula" which we review in the next section.

The overall plan of the paper is as follows. In $\S 3$ we state the fixed point formulae and use them to prove the rigidity of $d_{S}$. In $\S \S 4$ and 5 we combine these arguments with the power series $R_{q}$ and so interpret $\tau_{q}(M)$, the Chern character of index $\left(d_{S} \otimes R_{q}\right) \quad\left(=\operatorname{ch} \cdot \operatorname{index}\left(d_{S} \otimes R_{q}\right)\right)$, as a meromorphic function on a complex torus $T_{q^{2}}$ which has poles only at roots of 1 and which has no poles on a certain circle $S \subset T_{q^{2}} . \S \S 6$ and 7 then show that under the spin hypothesis, $\tau_{q}(M)$ has no poles anywhere on $T_{q^{2}}$, and hence is constant. In all these sections we assume isolated fixed points. Then, in $\S \S 8$ and 9 we extend these arguments to $S^{1}$ actions with larger fixed point sets. $\S 10$ deals with questions of orientability and brings a new proof of a theorem of Edmonds. In $\S 11$ we follow Witten to extend the rigidity to auxiliary spin-bundles $V$ on $M$, which approximate the tangent bundle $T$ of $M$ in the sense that $w_{2}(V-T)=0$ and $\frac{1}{2} p_{1}(V-T)=0$, with these classes now taken in the equivariant sense. Actually our conditions are slightly weaker than the ones formulated by Witten. 
In $\S 12$ we discuss the rigidity for $\not \partial \otimes R_{n}^{\prime}$. Finally in $\S 13$ we describe the modular properties of $\tau_{q}(M)$, and $\tau_{q}^{\prime}(M)=\mathrm{ch} \cdot \operatorname{index}\left(\not \partial \otimes R_{q}^{\prime}\right)$, and in $\S 14$ we state the "almost complex" version of the rigidity theorem, which can be treated in precisely the same manner. Because a version of this theorem was quite recently proved by $\mathrm{F}$. Hirzebruch $[\mathrm{H}]$ we did not go into the details there.

Our approach is in a sense "low-brow" and hopefully accessible to nonexperts. For a more lofty overview of the entire subject of elliptic cohomology we refer the reader to G. Segal's incisive Bourbaki account [S] as well as [La].

\section{THE FIXED POINT FORMULA FOR ELLIPTIC COMPLEXES}

Recall that the fixed point formula of [A-B] deals with the trace of a geometrically induced automorphism $f$ of an elliptic operator:

$$
D: \Gamma(E) \rightarrow \Gamma(F) \text {. }
$$

When $f$ has only nondegenerate fixed points, the result is of the following sort:

$$
\text { Trace } f \mid(\operatorname{ker} D-\operatorname{coker} D)=\sum_{p \in M: f(p)=p} \mu_{p}(D, f) .
$$

The "multiplicity" $\mu_{p}$ is locally determined by the behavior of $D$ and $f$ near the fixed point $p$.

For instance, if we are dealing with an even-dimensional, oriented manifold $M$ and an $S^{1}$ action on $M$ with only isolated fixed points, this formula easily specializes to yield the following.

Let $f$ denote the action of $e^{i \theta}$ in $S^{1}$. Also if $p$ is a fixed point of the action let

$$
T_{p}=E_{1} \oplus \cdots \oplus E_{d}, \quad d=\frac{1}{2} \operatorname{dim}_{\mathbf{R}} M,
$$

be a decomposition of the tangent space to $M$ at $p$ into $S^{1}$-invariant, real 2-planes. (On each 2-plane, $S^{1}$ is represented in a nontrivial manner, because $p$ is assumed to be isolated.)

We next choose an isomorphism of $\mathbf{C}$ with $E_{j}$ relative to which the representation of $S^{1}$ on $E_{j}$ is given by $e^{i \theta} \rightarrow e^{i m_{j} \theta}$ with $m_{j} \in \mathbf{Z}$. But we choose these orientations to be compatible with the orientation of $M, \mathrm{C}$ being oriented by $\{1, i\}$ in the usual manner. The resulting set of integers $m_{1}, \ldots, m_{d}$ is called the set of exponents of the action at $p$. Note that they are unique up to sign, but the signs of the $m_{i}$ can only be changed in even lots.

With this understood, and setting $\lambda=e^{i \theta}$, the fixed point formulae for our three complexes read:

$$
\text { trace } f \text { on index }\left(d+d^{*}\right)=\sum_{f(p)=p}+1,
$$




$$
\begin{aligned}
\text { trace } f \text { on index } d_{S} & =\sum_{f(p)=p} \prod_{j=1}^{d} \frac{\left(1+\lambda^{m_{j}}\right)}{\left(1-\lambda^{m_{j}}\right)}, \\
\text { trace } f \text { on index } \not \partial & =\sum_{f(p)=p} \prod_{j=1}^{d} \frac{\lambda^{m_{j} / 2}}{\left(1-\lambda^{m_{j}}\right)}
\end{aligned}
$$

As trace $f$ on $L^{n}$ is clearly $\lambda^{n}$ these formulae in principle determine the index $=$ ker-coker qua $S^{1}$ module in every case. In fact, the formulae go further. They immediately prove the universal rigidity for each of the three operators.

For (3.4) this is true by inspection: If the trace of multiplication by $e^{i \theta}$ is independent of $\theta$ then only the trivial representation occurs. For (3.5) we have to work a little harder and take the substitution $\lambda=e^{i \theta}$ more seriously: In terms of $\lambda$, the left-hand side (LHS), being the character of a finite-dimensional virtual $S^{1}$ module, is of the form

$$
\text { LHS }=\sum_{n=-N}^{n=+N} a_{n} \lambda^{n}
$$

This is the restriction to $S^{1} \subset \mathbf{C}$ of a finite Laurent series on the $\lambda$-plane. On the other hand, the right-hand side (RHS) is the restriction to $S^{1}$ of the rational function

$$
\mathrm{RHS}=\sum_{f(p)=p} \frac{\Pi\left(1+\lambda^{m_{j}}\right)}{\Pi\left(1-\lambda^{m_{j}}\right)}, \quad \lambda=e^{i \theta}
$$

also defined on the $\lambda$-plane.

The fixed point formula implies that these two expressions are equal on a dense set of the circle $|\lambda|=1$. Hence they are equal as rational functions on C. In fact, both obviously extend to rational functions on $S^{2}=\mathbf{C} \cup \infty$.

But the LHS can have poles only at 0 and at $\infty$ while the RHS has poles only on the unit circle. Since the LHS and RHS are equal, both LHS and RHS are constant as functions of $\lambda$, this constant being the signature $\tau(M)$. QED

Essentially the same argument holds for the Dirac operator (3.6). All one might have to do is to pass to a double cover of $S^{1}$ if the square root occurs on the RHS. (In fact, taking $\lambda=0$ or $\lambda=\infty$ in (3.6) shows that the index for the Dirac operator vanishes (as in [A-H]).) Thus for an $S^{1}$ action with only isolated fixed points, the rigidity properties of all our operators are beautifully transparent.

In the next sections we will present the extension of this rigidity argument to the family of operators $\left\{d_{S} \otimes R_{n}\right\}$, but still in the isolated singularity case.

\section{Proof of The RIGIDITY of $d_{S} \otimes R_{n}$; BEgINNINGS}

Recall that under a twist by $R$ the multiplicities of our fixed point formula behave very simply. At a fixed point $p$ of the action we have a natural lifting 
$l_{p}$ of $S^{1}$ to the fiber over $p, O_{p}$, of the orthogonal frame-bundle over $M$. In terms of $l_{p}$ the twisted multiplicity $\mu_{p}(D \otimes R)$ is related to the untwisted one $\mu_{p}(D)$ by

$$
\mu_{p}(D \otimes R)=\mu_{p}(D) \cdot \operatorname{ch~} l_{p}^{*} R .
$$

In short we simply multiply by the character of $R$ restricted to $l_{p} S^{1}$. For instance (in the notation of $\S 3$ )

$$
\mu_{p}\left(d_{S} \otimes T\right)=\prod \frac{\left(1+\lambda^{m_{i}}\right)}{\left(1-\lambda^{m_{i}}\right)} \cdot\left[\sum\left(\lambda^{m_{i}}+\lambda^{-m_{i}}\right)\right] .
$$

If one now attempted to prove the rigidity of $d_{S} \otimes T$ one would have to deal with this multiplicity in the corresponding fixed point formula:

$$
\sum_{n=-N}^{n=+N} a_{n} \lambda^{n}=\sum \mu_{p}
$$

But now the expression

$$
\frac{1+\lambda^{n}}{1-\lambda^{n}} \cdot\left(\lambda^{n}+\lambda^{-n}\right)
$$

and hence each $\mu_{p}$, has a pole at 0 and $\infty$ as well as on the unit circle $|\lambda|=1$. For this reason, the old argument does not prove the vanishing of $a_{n}$ 's; at best, it yields inequalities for the $N$ in (4.3).

It is important to note, however, that by comparing the right and left sides of (3.2) this argument does prove that the poles of $\mu_{p}$ on the unit circle must cancel in the RHS, just as before!

This, then, is the situation if one attempts to prove the rigidity of $d_{S} \otimes T$ in isolation.

Let us now turn to the Witten framework; we write down the fixed point formula for the twists $d_{S} \otimes R_{n}$ of (2.10) and then sum them to form the formal expression $\sum q^{n}$ index $\left(d_{S} \otimes R_{n}\right)$. Precisely let $a_{n m}$ be defined by

$$
\operatorname{index}\left(d_{S} \otimes R_{n}\right)=\sum_{m=-N_{n}}^{m=+N_{n}} a_{n m} L^{m}
$$

so that the sum in question is given by the expression

$$
\operatorname{index}\left(d_{S} \otimes R_{q}\right) \equiv \sum_{n} \sum_{m} q^{n} a_{n m} L^{m} .
$$

The LHS of the fixed point formula is now given by

$$
\mathrm{ch} \cdot \operatorname{index}\left(d_{S} \otimes R_{q}\right)=\sum_{n, m} q^{n} a_{n}(\lambda),
$$

where $a_{n}(\lambda)$ denotes the finite Laurent series

$$
a_{n}(\lambda)=\sum_{m} a_{n m} \lambda^{m}
$$


We turn now to the RHS of the fixed point formula, that is, to the multiplicity formulae $\mu_{p}\left(d_{S} \otimes R_{n}\right)$ and to the power series

$$
\mu_{p}(q ; \lambda)=\sum q^{n} \mu_{p}\left(d_{S} \otimes R_{n}\right),
$$

a formal power series in $q$ with coefficients which are meromorphic functions in $\lambda$.

Using the well-known identity, qua formal power series in $q$,

$$
S_{q}(T)=\frac{1}{\Lambda_{-q}(T)}
$$

we can recast Witten's $R_{q}$ into the form

$$
R_{q}(T)=\bigotimes_{k=1}^{\infty} \Lambda_{q^{k}}(T) / \Lambda_{-q^{k}}(T)
$$

Then, using the multiplicative properties of $\Lambda$ we arrive at the formula

$$
\mu_{p}\left(d_{S} \otimes R_{q}\right)=\prod_{i=1}^{d} \frac{\left(1+\lambda^{m_{i}}\right)}{\left(1-\lambda^{m_{i}}\right)} \prod_{k=1}^{\infty} \frac{\left(1+q^{k} \lambda^{m_{i}}\right)\left(1+q^{k} \lambda^{-m_{i}}\right)}{\left(1-q^{k} \lambda^{m_{i}}\right)\left(1-q^{k} \lambda^{-m_{i}}\right)},
$$

where the right-hand side is taken in the sense of a formal power series in $q$. It clearly makes sense as such; in fact, it is of the form

$$
\text { RHS }=\sum b_{n}^{p}(\lambda) q^{n}
$$

where $b_{n}^{p}(\lambda)$ is a rational function

$$
b_{n}^{p}(\lambda)=\mu_{p}\left(d_{S} \otimes R_{n}\right) .
$$

With all this understood, the fixed point formula applied to all the $d_{S} \otimes R_{n}$ is summarized by the equality

$$
\sum_{n} a_{n}(\lambda) q^{n}=\sum_{n}\left\{\sum_{\{p\}} b_{n}^{p}(\lambda) q^{n}\right\}
$$

Note that it is immediate from the preceding equality that the sum over the fuxed points, $\sum_{\{p\}} b_{n}^{p}(\lambda)$, has no poles on $|\lambda|=1$.

Further progress is made by exploiting the modular properties of $\mu_{p}\left(d_{S} \otimes R_{q}\right)$ and it will be in the language of elliptic functions that the proof of the rigidity will be carried out in the next sections.

\section{THE MULTIPLICITY AS A MEROMORPHIC FUNCTION ON $\mathbf{C}^{*} / q^{2}$}

If one divides the multiplicative group of nonzero complex numbers by the subgroup generated by an element $q \neq 0$, one obtains a complex torus, which we denote by $T_{q}$.

Our first remark will be that the formal series (4.12) is a convergent Laurent series in the region $0<|q|<1$, and $|q|^{1 / N}<|\lambda|<|q|^{-1 / N}$ of the pull-back to 
$\mathbf{C}^{*}$ of a meromorphic function on the torus $T_{q^{2}}$. Here $N$ is some (determined) positive integer.

To explain this, it is best to start with the function

$$
\varphi_{0}(\lambda)=\frac{1+\lambda}{1-\lambda} \text { on } \mathbf{C}^{*}
$$

and consider a corresponding infinite product

$$
\varphi_{1}(\lambda)=\prod_{k=1}^{\infty}\left(\frac{1+q^{k} \lambda}{1-q^{k} \lambda}\right)\left(\frac{1+q^{k} \lambda^{-1}}{1-q^{k} \lambda^{-1}}\right) .
$$

This product converges absolutely in the annulus $D:|q|<|\lambda|<\frac{1}{|q|} ;|q|<1$.

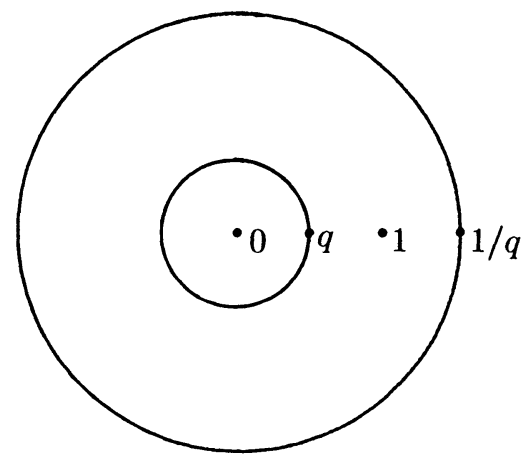

Figure 1

Indeed, in this region $\left|q^{k} \lambda\right|$ and $\left|q^{k} \lambda^{-1}\right|$ are $\leq|q|^{k-1}$ and $|q|<1$. Thus $\varphi_{1}(\lambda)$ is a holomorphic function on the annulus $D$; and

$$
\varphi(\lambda) \equiv \varphi_{0}(\lambda) \cdot \varphi_{1}(\lambda)
$$

is therefore a meromorphic function on $D$ with a pole of order 1 at $\lambda=1$.

Let us now make the substitution $\lambda \longrightarrow \lambda \cdot q$. Formally,

$$
\varphi(\lambda q)=-\varphi(\lambda) .
$$

Indeed, under this substitution $\left(1+q^{k} \lambda\right)$ moves to $\left(1+q^{k+1} \lambda\right)$ and $\left(1+q^{k} \lambda^{-1}\right)$ to $\left(1+q^{k-1} \lambda^{-1}\right)$ so

$$
\varphi(\lambda q)=\frac{1-\lambda}{1-\lambda^{-1}} \cdot \frac{1+\lambda^{-1}}{1+\lambda} \cdot \varphi(\lambda)=-\varphi(\lambda) .
$$

As this substitution maps $|q|<|\lambda|<1$ to $1<|\lambda|<\frac{1}{|q|}$, we see that this symmetry can be used to extend $\varphi(\lambda)$ to a meromorphic function on all of $\mathbf{C}^{*}$. We write $\varphi(\lambda)$ for this extended function also. In view of the symmetry $\varphi(q \lambda)=-\varphi(\lambda)$, we then have

$$
\varphi\left(q^{2} \lambda\right)=\varphi(\lambda)
$$


It follows that $\varphi$ is the pull-back of a meromorphic function (also written $\varphi$ ) on the torus $T_{q^{2}}$. As such $\varphi$ clearly has a pole of order 1 at the cosets of 0 and $q \bmod q^{2}$, and a zero of order 1 at the cosets of -1 and $-q$. Now the points $\pm q$ and \pm 1 are precisely the points of order 2 on $T_{q^{2}}$, so that up to a normalization factor $\varphi$ is one of the canonically associated meromorphic functions on the complex torus $T$ which have poles of order one at the origin and at one of the other points, $\omega_{1}$, of order 2 on $T$ while vanishing with order one at the remaining two points of order 2 , say $\omega_{2}$ and $\omega_{3}$.

In the more standard picture of an additive torus, the divisor of $\varphi$ is of the form

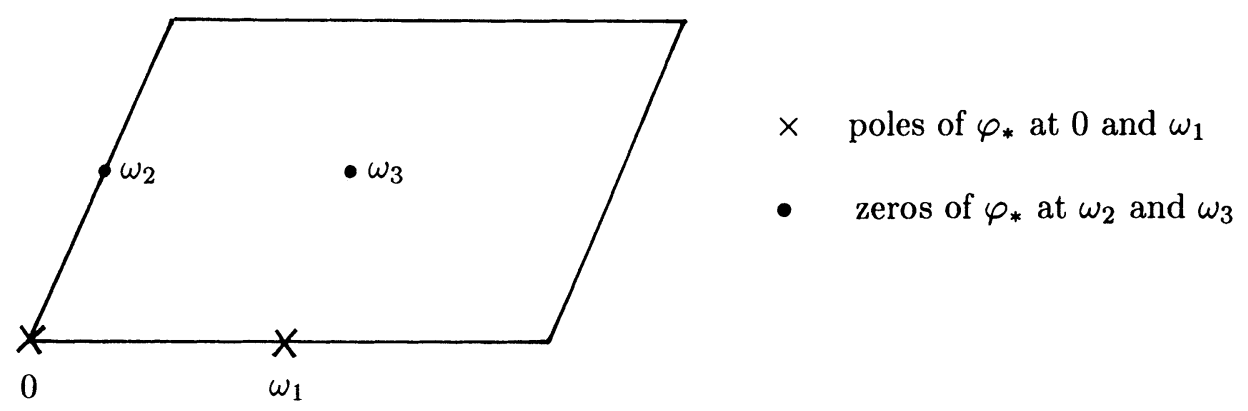

FIGURE 2

Note also that on $T_{q^{2}}$ the residue of $\varphi$ at the identity is given by

$$
\left.\operatorname{res} \varphi\right|_{\lambda=1}=-2 \frac{\Pi\left(1+q^{n}\right)^{2}}{\Pi\left(1-q^{n}\right)^{2}} .
$$

Hence the renormalized $\varphi$ given by

$$
\varphi_{*}=\frac{1}{2} \frac{\Pi\left(1-q^{n}\right)^{2}}{\Pi\left(1+q^{n}\right)^{2}} \cdot \varphi
$$

has residue -1 at 1 , and, therefore, +1 at $q$.

The canonical nature of this function on all tori $\left(T, \omega_{1}\right)$ with a singled out point $\omega_{1}$ with $\omega_{1}+\omega_{1}=0, \omega_{1} \neq 0$ (with $T$ additive as in Figure 2), will be used later to discuss the modular properties of index $\left(d_{S} \otimes R_{q}\right)$.

To return to the multiplicity formula (4.12), observe that the function $\varphi_{0}(\lambda)$ $=(1+\lambda) /(1-\lambda)$ is the prototype for all the factors of $\mu_{p}$. In fact, if $\psi_{m}$ denotes the operation of raising $\lambda$ to the $m$ th power, then at a fixed point $p$ of our circle action, with exponents $m_{1}, \cdots, m_{d}$, the multiplicity $\mu_{p}$ has the form

$$
\mu_{p}=\psi_{m_{1}} \varphi \cdot \psi_{m_{2}} \varphi \cdots \psi_{m_{d}} \varphi
$$


Because the $n$th power operation $\psi_{n}$ is also well defined on $T_{q^{2}}$ and commutes with the pullback, our discussion concerning $\varphi$ identifies $\mu_{p}\left(d_{S} \otimes R_{q}\right)$ as a Laurent series of the meromorphic function $\psi_{m_{1}} \varphi \cdots \psi_{m_{d}} \varphi$ on $T_{q^{2}}$ :

$$
\mu_{p}\left(d_{S} \otimes R_{q}\right)=\psi_{m_{1}} \varphi \cdots \psi_{m_{d}} \varphi
$$

At this stage we can assemble these remarks to yield a first interesting consequence of the fixed point theorem.

Proposition 5.1. Consider an action of $S^{1}$ by isometries on the oriented compact Riemannian manifold $M$ with isolated fixed points, and let $N$ be the largest of the exponents $\left\{m_{i}\right\}$ occurring at them. Then the formal series

$$
\operatorname{ch} \cdot \operatorname{index}\left(d_{S} \otimes R_{q}\right)=\sum q^{n} a_{n m} \lambda^{m}
$$

is convergent in the annulus $0<|q|<1,|q|^{1 / N}<\lambda<|q|^{-1 / N}$ to the Laurent series of a meromorphic function on the torus $T_{q^{2}}$.

Proof. Recall that $\varphi$ is the product of a $q$-independent rational function of $\lambda$ with poles only on the circle $|\lambda|=1$ and a convergent Laurent expansion in $q$ and $\lambda$ with domain of convergence $0<|q|<1$ and $|q|<|\lambda|<|q|^{-1}$. Thus, $\psi_{m} \varphi$ is the product of a $q$-independent, rational function of $\lambda$ with poles only on $|\lambda|=1$ and a convergent Laurent expansion in $q$ and $\lambda$ whose domain of convergence has shrunk to $|q|^{1 / m}<\lambda<|q|^{-1 / m}$. Hence, by (5.8) each multiplicity $\mu_{p}\left(d_{S} \otimes R_{q}\right)$ is the product of a $q$-independent, rational function on $\mathbf{C}$ with poles only on the circle $|\lambda|=1$ with a convergent Laurent series on the region $|q|^{1 / N}<|\lambda|<|q|^{-1 / N}$. Also, $\mu_{p}\left(d_{S} \otimes R_{q}\right)$ is the pull-back to $\mathbf{C}^{*}$ of a meromorphic function on $T_{q^{2}}$.

By the fixed point theorem, we have, as was already remarked in $\S 4$, the equality

$$
\operatorname{ch} \cdot \operatorname{index}\left(d_{S} \otimes R_{q}\right) \equiv \sum_{p} \mu_{p}\left(d_{S} \otimes R_{q}\right),
$$

qua formal powers series in $q$ with coefficients which are the restriction to the circle $|\lambda|=1$ of rational functions on the $\lambda$-plane. By (4.14), the polar parts of the coefficients of the formal $q$-expansion of the RHS in (5.11) cancel on the circle $|\lambda|=1$. Thus, the RHS of (5.11) defines a convergent Laurent expansion in $q$ and $\lambda$ with domain $0<|q|<1$ and $|q|^{1 / N}<|\lambda|<|q|^{-1 / N}$, the only poles possible lie on $|\lambda|=1$, and (4.14) implies that there are none. To see this, we write $\mu_{p}=r_{p}(\lambda) \cdot h_{p}(\lambda, q)$, where $r_{p}$ is a rational function on $\mathbf{C}$ with its poles on $S$, and $h_{p}$ is a function on $\lambda$ and $q$ with a convergent power series in $\lambda$ and $q$ for $|q|<1$ and $\lambda$ in an annulus that contains $S$. Thus, a pole in $\sum_{p} \mu_{p}$ at $c \in S$ of order $N>0$ has leading coefficient a function $f_{N}(q)$, which is a linear combination of the values of the $\left\{h_{p}\right\}$ and their $\lambda$-derivatives at $c$ to some finite order-hence, an analytic function of $q$ for $|q|<1$. So taking the polar part and taking the $q$-expansion are commuting operations 
on $\sum_{p} \mu_{p}$. Thus, the index theorem plus the fixed point formula applied to each $\left\{d_{S} \otimes R_{n}\right\}_{n \geq 0}$ insures that the power series expansion of $f_{N}$ is zero, and $f_{N} \equiv 0$.

Thus, (5.11) identifies the LHS as the restriction to the circle $|\lambda|=1$ of a convergent power series in $q$ and Laurent series in $\lambda$ on the domain $0<|q|<$ $1,|q|^{1 / N}<|\lambda|<|q|^{-1 / N}$. This convergent Laurent expansion represents, of course, a meromorphic function of the type described because the RHS is a sum of such functions. QED

Remarks. In view of this proposition the character of the index of $d_{S} \otimes R_{q}$ is a well-defined meromorphic function on $T_{q^{2}}$, which we call the $q$-signature of $M$, and denote by $\tau_{q}(M)$

$$
\tau_{q}(M) \underset{\text { def }}{\equiv} \operatorname{ch} \cdot \text { index }\left(d_{S} \otimes R_{q}\right)
$$

In some formal sense $\tau_{q}(M)$ represents the "signature" of the loop-space $\mathscr{L}(M)$. But in any case it is a well-defined meromorphic function on $T_{q^{2}}$ and has no poles on the circle $S \subset T_{q^{2}}$ corresponding to $|\lambda|=1$.

In this context the rigidity of $d_{S} \otimes R_{q}$ is equivalent to the assertion that $\tau_{q}(M)$ has no poles at all on $T_{q^{2}}$.

A priori $\tau_{q}(M)=\sum \mu_{p}\left(d_{S} \otimes R_{q}\right)$ has only poles at the points of finite order on $T_{q^{2}}$ because the multiplicities $\mu_{p}$ have only poles there. Hence one can test for the poles of $\tau_{q}(M)$ by considering the behavior of its translates $t_{\alpha} \tau_{q}(M)$ by $n$th roots of 1 on $T_{q^{2}}$. For example, consider the points of order 2 first. For $\alpha=-1, t_{\alpha}$ preserves $S$, and therefore $t_{\alpha} \tau_{q}(M)$ has no poles on $S$ because $\tau_{q}(M)$ did not to start with.

Next set $\alpha=q$, the more interesting point of order 2 on $T_{q^{2}}$. Then

$$
t_{\alpha} \varphi=-\varphi
$$

hence at a fixed point $p$ with exponents $m_{1}, \cdots, m_{d}$ we obtain

$$
t_{\alpha} \mu_{p}=(-1)^{\sum m_{j}} \cdot \mu_{p}
$$

If the exponents of the action satisfy the condition

$$
\sum m_{j}^{(p)} \equiv \sum m_{i}^{\left(p^{\prime}\right)}(\bmod 2)
$$

for any pair of fixed points $p$ and $p^{\prime}$, then $t_{\alpha} \tau_{q}(M)= \pm \tau_{q}(M)$ and $\tau_{q}(M)$ also has no poles on $q \cdot S$. In short, under the exponent condition $(5.15) \tau_{q}(M)$ has no poles of order 2 .

We will show later that (5.15) is always satisfied on a spin manifold (this assertion is also made in $[\mathrm{A}-\mathrm{H}])$. But it is easily seen to fail for other actions. For example, consider the projective space $P(V)$ of lines in the $S^{1}$ module

$$
V=L^{0} \oplus L^{1} \oplus L^{2}
$$


Then $P(V)$ is $\mathrm{C} P^{2}$ with an induced holomorphic $S^{1}$-action whose fixed points correspond precisely to the axes $L^{0}, L^{1}$, and $L^{2}$ of $V$. At these fixed points, the exponents are seen to be

$$
\begin{array}{rrr}
L^{0} & 1 & 2 \\
L^{1} & -1 & 1 \\
L^{2} & -2 & -1
\end{array}
$$

where we have now chosen the holomorphic orientations at the fixed points. Note that the parities of their sums are different at $L^{1}$ and $L^{2}$.

For this example we have

$$
\tau_{q}=2 \varphi \cdot \psi_{2} \varphi-\varphi^{2}
$$

and it is easy to check that this $\tau_{q}$ has no pole at 1 , but does have a pole of order two at $q$. Of course this is not alarming because $\mathbf{C} P^{2}$ is not a spin manifold.

To test for poles of $\tau_{q}(M)$ at higher roots of 1 is more difficult and requires a "transfer" argument as well as a twisted form of Proposition 5.1. We explain this extension in the next section.

\section{Twisting $\tau_{q}$}

The arguments of the preceding sections admit some straightforward extensions which correspond in the present context to the operation of twisting $d_{S}$ by an auxiliary complex $S^{1}$-bundle $F$ over $M$. At each fixed point $p$, the fiber $F_{p}$ of $F$ at $p$, decomposes into a sum

$$
F_{p}=\bigoplus_{j} L^{m_{j}}
$$

of irreducible $S^{1}$ modules and we call the integers $m_{j}$ the exponents of $F$ at $p$.

The fixed point formula for the twist of $d_{S}$ by $F$ then reads

$$
\operatorname{ch} \cdot \operatorname{index}\left(d_{S} \otimes F\right)=\sum \mu_{p}\left(d_{S}\right) \cdot \operatorname{ch}\left(F_{p}\right)
$$

with ch $F_{p}=\sum \lambda^{m_{j}}$. Just as before, this relation implies that on the unit circle the poles of the right-hand side cancel out.

Such a result persists if we twist $d_{S} \otimes R_{q}$ by $F$ but would destroy the elliptic nature of the answer. To remedy this we will twist $d_{S} \otimes R_{q}$ by a $q$-dependent vector bundle constructed as follows. Recall the Laurent expansion of our basic meromorphic function, $\varphi$, on $T_{q^{2}}$ :

$$
\varphi(\lambda)=\frac{1+\lambda}{1-\lambda} \cdot \prod_{n=1}^{\infty} \frac{1+q^{n} \lambda}{1-q^{n} \lambda} \prod_{n=1}^{\infty} \frac{1+q^{n} \lambda^{-1}}{1-q^{n} \lambda^{-1}}
$$

and let $a$ be a point in the region, $|q|<|\lambda|<1$, of convergence of the infinite product. 
It then follows that the translate by $a$ of $\varphi$ defined by

$$
\varphi_{a}(\lambda) \equiv t_{a} \varphi(\lambda) \equiv \varphi(a \lambda)
$$

is the product of a $q$-independent rational function with simple pole at $\lambda=1 / a$ and a Laurent series in $q$ and $\lambda$ which converges for $0<|q|<1$ on the annulus $|q / a|<|\lambda|<1 /|a q|$ containing the unit circle. Notice that the Laurent expansion is of "finite type in $\lambda$." That is, the expansion has the form

$$
\sum_{n=0}^{\infty} q^{n} b_{n}(\lambda)
$$

with each $b_{n}(\lambda)$ a finite Laurent series. Indeed, this property of (5.2) is not altered by the translation by $a$.

Since $\varphi(\lambda q)=-\varphi(\lambda)$, equation (6.4) defines the translate $\varphi_{a}(\lambda)$ for any point $a \in T_{q^{2}}$. This function $\varphi_{a}(\lambda)$ is then a meromorphic function on $T_{q^{2}}$.

It follows by the splitting principle that $\varphi_{a}$ can be extended to complex vector bundles $F$, to yield a formal power series

$$
\varphi_{a}(F)=\sum_{n=0}^{\infty} q^{n} b_{n}(F) .
$$

In fact, for $|q|<|a|<1$, we have the expansion

$$
\varphi_{a}(F)=\frac{\Lambda_{a} F}{\Lambda_{-a} F} \bigotimes_{n=1}^{\infty}\left(\frac{\Lambda_{a q^{n}} F}{\Lambda_{-a q^{n}} F} \otimes \frac{\Lambda_{a^{-1} q^{n}} F^{*}}{\Lambda_{-a^{-1} q^{n}} F^{*}}\right)
$$

for the bundle analogue to (6.3); here, $F^{*}$ is the complex, dual bundle to $F$.

Now, then, the arguments of the previous sections easily extend to the twisting of $d_{S} \otimes R_{q}$ by $\varphi_{a}(F)$ to yield the following.

Proposition 6.1. In the context of our discussion above, define $\tau_{q}\left(M ; \varphi_{a}(F)\right)$ as the formal Laurent series

$$
\tau_{q}\left(M ; \varphi_{a}(F)\right) \equiv \mathrm{ch} \cdot \operatorname{index}\left(d_{S} \otimes R_{q} \otimes \varphi_{a}(F)\right) .
$$

Then $\tau_{q}\left(M ; \varphi_{a}(F)\right)$ converges on some annulus $D$ containing $S=(|\lambda|=1)$ to the Laurent series of a meromorphic function on $T_{q^{2}}$ which has no poles on the unit circle $S \subset T_{q^{2}}$. Similarly twisting by a finite number of $\varphi_{a}(F)$ 's preserves the regularity of $\tau_{q}$ on $S$.

As an example consider the action of (5.16) on the "subbundle $H^{*}$ " of $P(V)$ whose fiber at a line $L$ is $L$ itself. The exponents of $H^{*}$ are then 0,1 , and 2 , respectively, so that

$$
\tau_{q}\left(M ; \varphi_{a}\left(H^{*}\right)\right)=\varphi \cdot \psi_{2} \varphi \cdot \psi_{0} \varphi_{a}-\varphi \cdot \varphi \cdot \varphi_{a}+\psi_{2} \varphi \cdot \varphi \cdot \psi_{2} \varphi_{a} .
$$

Thus Proposition 6.1 asserts that this rational function on $T_{q^{2}}$ has no pole on $S$. This can be checked from first principles using the polar properties of $\varphi$, but already requires a more subtle analysis than the regularity of $\tau_{q}(M)$ (as given by (5.18)) required. 
Remarks. In general, $\varphi_{a}(F) \neq \varphi_{a}\left(F^{*}\right)$. Hence $\varphi_{a}$ cannot be extended to an operation on real vector bundles. Note, however, that $\varphi_{ \pm q^{1 / 2}}(\lambda)$ is symmetric in $\lambda$ and $\lambda^{-1}$, and so extends to a well-defined operation on real vector bundles: If $F$ is a real vector bundle, we have

$$
\varphi_{ \pm q^{1 / 2}}(F) \equiv \frac{\bigotimes_{n=1}^{\infty} \Lambda_{ \pm q^{n-1 / 2}} F}{\bigotimes_{n=1}^{\infty} \Lambda_{\mp q^{n-1 / 2}} F} .
$$

\section{THE TRANSFER FORMULAE}

To prove the rigidity of $\tau_{q}(M)$, we plan to show that under suitable circumstances none of the translates $t_{a} \tau_{q}(M), a \in T_{q^{2}}$, by points of finite order on $T_{q^{2}}$, has a pole on the circle $S \subset T_{q^{2}}$. This will follow immediately from the discussion of the previous section if we can express these $t_{a} \tau_{q}(M)$ as twisted forms of $\tau_{q}$ on some auxiliary manifolds. It should also be clear that it is sufficient to carry out this program as $k$ ranges over the positive integers and as $a$ ranges over the roots of the form

$$
a=\alpha^{s}, \quad \alpha^{k}=q
$$

for $k$ and $s$ having no common factors save 1 .

The auxiliary manifold pertinent to the translation by such an $a$ is the manifold $M_{k}$ of fixed points in $M$ under the action of the subgroup $\mathrm{Z}_{k} \subset S^{1}$, generated by $\xi \equiv e^{2 \pi i / k}$. Thus,

$$
M_{k}=\text { fixed point set of } \mathbf{Z}_{k} \text {. }
$$

The cyclic group $Z_{k}$ acts on the normal bundle of $M_{k}$ in $M$ and we have a natural decomposition of the tangent space $T$ of $M$ along $M_{k}$ according to the irreducible real representations $\{\rho\}$ of $\mathbf{Z}_{k}$ :

$$
T \mid M_{k}=\sum T_{\rho} .
$$

Now, our $S^{1}$-action on $M$ induces an action on $M_{k}$; and its differential induces an action on $T \mid M_{k}$ which preserves the decomposition (7.3) thus making each $T_{\rho}$ an $S^{1}$-bundle over $M_{k}$.

To illustrate our plan in the simplest instance, assume that $k$ is odd. In this case, the irreducible real representations $\rho$ of $\mathbf{Z}_{k}$, other than the trivial one, admit unique complex structures so that $\xi$ maps to $\xi^{r}$ under $\rho$, with $r=1, \ldots,(k-1) / 2$.

Hence, in our context, each $T_{\rho}$ for $\rho$ not trivial can be given a unique complex structure on which $\xi$ acts by $\xi^{r}$ for some $r=1, \ldots,(k-1) / 2$; and let $T_{r}$ denote this complex bundle. Then, the decomposition in (7.3) has the form

$$
T \mid M_{k}=T M_{k}+T_{1}^{\#}+\cdots+T_{(k-1) / 2}^{\#}
$$


with \# indicating the underlying real bundle of the indicated complex one.

The bundle $T M_{k}$ is the tangent bundle to $M_{k}$ and corresponds to the trivial representation. It need not carry a complex structure, but if we use the natural (complex) orientation of $T_{r}^{\#}$, then (7.4) does serve to induce an orientation on $T M_{k}$, and hence on $M_{k}$, once one is selected for $M$ and hence for $T$.

With all this understood, assuming now that $M$ is a spin manifold, we have the following transfer formula for odd $k$ :

$$
t_{\alpha^{s}} \tau_{q}(M)=\tau_{q}\left(M_{k}^{\prime} ; \bigotimes_{r=1}^{(k-1) / 2} \varphi_{\alpha^{(\omega r)}}\left(T_{r}\right)\right),
$$

where $\omega(r) \in\{1, \cdots, k-1\}$ is the $\bmod k$ reduction of $s \cdot r$ and where $M_{k}^{\prime}$ denotes $M_{k}$, but with a specific orientation which may differ from the induced one. In fact, if the connected components of $M_{k}$ are labelled by $\left\{M_{k, \sigma}\right\}$, and if the induced orientation is denoted by $+1 \in\{ \pm 1\}$, then the orientation on $M_{k, \sigma}^{\prime}$ is given by $(-1)^{u(k, \sigma)}$ where $u(k, \sigma)$ is computed from the exponents at any fixed point $p \in M_{k, \sigma}$. It is the number $\varepsilon(p)$ in (7.9) and Lemma 7.1 below. If $S^{1}$ acts on a component of $M_{k}$ with no fixed points, then that component contributes 0 to (7.5) and any orientation used for that component will suffice.

To prove (7.5), we can apply the fixed point formula to both sides and compare the contributions at each fixed point $p$. Indeed, partition the exponents at $p$ into the various exponents of $T_{r}^{\#}$ and $T M_{k}$, with the exponents $\equiv \pm r$ $(\bmod k)$ occurring in $T_{r}^{\#}$. Using the complex orientation on $T_{r}^{\#}$ amounts to choosing $m_{j}<0$ if $\left|m_{j}\right|=k-r(\bmod k)$ and $m_{j}>0$ if $\left|m_{j}\right|=r(\bmod$ $k$ ). Let this be done and choose the remaining ones to be compatible with the induced orientation of $T M_{k}$. These are the $m_{j}$ which are congruent to $0 \mathrm{mod}$ $k$.

With this choice of $m_{j}$, the fixed point contribution to $\tau_{q}(M)$ is given by

$$
\mu_{p}=\prod_{j=1}^{d} \psi_{m_{j}} \varphi
$$

Hence $t_{\alpha^{s}} \mu_{p}=\prod t_{\alpha^{s}}\left(\psi_{m_{j}} \varphi\right)$. But,

$$
t_{\alpha^{s}} \psi_{m}=\psi_{m} t_{\alpha^{s}} \cdot m
$$

so that

$$
t_{\alpha^{\prime}} \mu_{p}=\prod_{j=1}^{d} \psi_{m_{j}} \varphi_{\alpha^{s m_{j}}}
$$

Now write $s \cdot m_{j}=\ell_{j} \cdot k+\omega_{j}$, where $\omega_{j} \in\{0, \cdots, k-1\}$ is the $\bmod k$ reduction of $s \cdot m_{j}$, and then introduce the integer

$$
\varepsilon(p)=\sum_{j=1}^{d} \ell_{j} .
$$


Then use the formula $\varphi_{q}=-\varphi$ to rewrite (7.8) in the form

$$
t_{\alpha^{s}} \mu_{p}=(-1)^{\varepsilon(p)} \prod_{j=1}^{d} \psi_{m_{j}} \varphi_{\alpha^{\left(\omega_{j}\right.}} .
$$

On the other hand, if $M_{k}$ replaces $M_{k}^{\prime}$ in the right side of (7.5), then this side contributes at a fixed point $p$ with this same expression, but with no factor $(-1)^{\varepsilon(p)}$. Indeed, the right side of (7.5) with $M_{k}$ replacing $M_{k}^{\prime}$ gives (7.10) without $(-1)^{\varepsilon(p)}$ and bracketed according to the congruences of the $m_{j}$. The $m_{j} \equiv 0(\bmod k)$ occur in $\tau_{q}\left(M_{k}\right)$, those with $m_{j}=1(\bmod k)$ occur in $\varphi_{\left(x^{* u 11}\right)}\left(T_{1}\right)$, and so on.

Thus, the transfer formula holds for odd $k$ once we prove that $(-1)^{\varepsilon(p)}=$ $(-1)^{\varepsilon\left(p^{\prime}\right)}$ when $p$ and $p^{\prime}$ are in the same component of $M_{k}$. But this assertion follows from the following more general fact, which is proved in $\S 9$.

Lemma 7.1. Suppose that $M$ is an oriented spin manifold on which $S^{1}$ acts with isolated fixed points, and, for $k \in \mathbf{N}$, let $M_{k} \subseteq M$ be a connected, oriented component of the fixed point set of $\mathbf{Z}_{k} \subset S^{1}$. Also, let $s \in \mathbf{Z}$ be relatively prime to $k$. Suppose that $p \in M_{k}$ is fixed by $S^{1}$ and that exponents $\left\{m_{j}\right\}$ for $\left.T\right|_{p}$ have been chosen to be compatible with the orientation of $M$, and also so that those which are $0 \bmod k$ give the correct orientation for $\left.T M_{k}\right|_{p}$. For each $m_{j}$, define $\ell_{j} \in \mathbf{Z}$ and $\omega_{j} \in\{0, \ldots, k-1\}$ by $s \cdot m_{j}=\ell_{j} \cdot k+\omega_{j}$, and then define $\varepsilon(p)=\sum_{j} \ell_{j}$. If $p, p^{\prime} \in M_{k}$, then $(-1)^{\varepsilon(p)}=(-1)^{\varepsilon\left(p^{\prime}\right)}$.

Before continuing, we note that changing $m_{j}$ to $-m_{j}$ does not change $(-1)^{\varepsilon(p)}$ if $m_{j} \equiv 0 \bmod (k)$, but $(-1)^{\varepsilon(p)}$ changes to $(-1)^{\varepsilon(p)+1}$ if $m_{j} \not \equiv 0$ $\bmod (k)$.

Assuming Lemma 7.1, there still remains the case $k$ even. In this case,

$$
\left.T\right|_{M_{k}}=T M_{k} \oplus T_{1}^{\#} \oplus \cdots \oplus T_{k / 2-1}^{\#} \oplus T_{k / 2}
$$

where each $T_{r}^{\#}$ for $r=1, \cdots, \frac{k}{2}-1$ is a real bundle which has a natural complex structure so that $\xi \in \mathbf{Z}_{k}$ acts on the resulting complex bundle, $T_{r}$, as $\xi^{r}$. The vector bundle $T_{k / 2}$ is a real bundle on which $\xi$ acts as $(-1)$.

A transfer formula such as (7.5) for the $k$ even case requires, a priori, that $M_{k}$ be orientable (so a signature operator can be defined). This is the case if $M$ is spin, a corollary of a general theorem of Edmonds, but we will bring a short proof of this assertion in $\S 10$. Here, we record this fact as

Lemma 7.2. If $M$ is spin and orientable, and if $S^{1}$ acts, then the fixed point set $M_{k}$ of the $\mathrm{Z}_{k} \subset S^{1}$ action is orientable.

Armed with this result, and again assuming that $M$ is spin, we can state the $k$ even transfer formula:

$$
t_{r^{s}} \tau_{q}(M)=\tau_{q}\left(M_{k}^{\prime} ; \bigotimes_{r=1}^{k / 2} \varphi_{\alpha^{(v(r)}}\left(T_{r}\right)\right)
$$


where, as in (7.5), $\omega(r)$ is the $\bmod k$ reduction of $s \cdot r$ and $M_{k}^{\prime}$ is $M_{k}$ with a specific orientation selected. To explain this orientation, we note first that the natural complex structures on $T_{r}^{\#}$ for $1 \leq r \leq \frac{k}{2}-1$ orient these vector bundles, and so, with the orientation of $T$, the Whitney sum $T M_{k} \oplus T_{k / 2}$ gets an orientation. Choose an orientation for $T M_{k}$ and call it +1 . With respect to this orientation, the orientation for $M_{k}^{\prime}$ is defined on a component, $M_{k, \sigma}$, to be $(-1)^{u(k, \sigma)}$ where $u(k, \sigma) \equiv \varepsilon(p)$ of Lemma 7.1 for any fixed point $p \in M_{k, \sigma}$. Here, a comment is in order. If the orientation of $M_{k}$ is changed, then $(-1)^{\varepsilon(p)}$ as computed in Lemma 7.1 also changes sign. This is because the orientation of $T_{k / 2}$ must change. To see why such a change affects $(-1)^{\varepsilon(p)}$, choose the signs for the exponents of $T$ at $p$ by the following convention.

The exponents which are not congruent to 0 or $\frac{k}{2} \bmod k$ are chosen as before. For the rest, fix an orientation for $T M_{k}$. This induces one on $T_{k / 2}$. Choose the signs of the exponents that are $0 \bmod k$ so that the orientations for $T M_{k}$ and for $T_{k / 2}$ at $p$ are separately compatible.

Now observe that a change in the orientation of $T_{k / 2}$ will require an odd number of exponents $m_{j}$ congruent to $\frac{k}{2} \bmod k$ to change sign, and this will change an odd number of the corresponding $\ell_{j}$ to $-\left(\ell_{j}+1\right)$.

With the preceding convention for the exponents at $p$ chosen, the argument for (7.12) is virtually identical to the one for (7.5), and so the transfer formula for both even and odd cases stands with Lemma 7.1, the subject of $\S 9$.

This brings us to the result we wanted.

Theorem 7.3. Let $S^{1}$ act on a compact, oriented spin manifold so as to have only isolated fixed points. Then its $q$-signature, $\tau_{q}$, has no poles on $T_{q^{2}}$ and, hence, is a constant. As a consequence, the rigidity theorem holds.

\section{FIXED POINT SETS OF HIGHER DIMENSION}

In general, the fixed points of an $S^{1}$ action on $M$ fall into components $\{P\}$ which are themselves smooth manifolds and although a fixed point formula is still valid, the contribution of each $P$ is now more complicated. The precise nature of this contribution is described by the $G$-signature theorem of AtiyahSegal [A-S].

We will here first formulate this result in purely $K$-theoretic terms which fit most naturally into our context. We will also spell out some of the $K$-theoretic details to make the arguments accessible to nonexperts.

First note that if $P$ is a component of the fixed point set of an $S^{1}$-action on the oriented manifold $M$, then along $P$ the tangent bundle $T$ of $M$ splits into a direct sum

$$
T \mid P=T P \oplus \bigoplus_{i=1}^{k} E_{i}^{\#}
$$


where $E_{i}^{\#}$ denotes the underlying real bundle or "realization" of the complex bundle $E_{i}$ on which $S^{1}$ acts by sending $\xi$ to $\xi^{m_{i}}$. Here, $\left|m_{i}\right| \neq\left|m_{j}\right|$ unless $i=j$. Although $E_{i}^{\#}$ is canonical, $E_{i}$ is not. Indeed, the complex conjugate $\bar{E}_{i}$ of $E_{i}$, of course, has the same "realization" $\bar{E}_{i}^{\#}=E_{i}^{\#}$, but the action $S^{1}$ on $\bar{E}_{i}$ is given by $\xi \rightarrow \xi^{-m_{i}}$. Hence the exponents are only canonical up to signs and a choice of the sign of $m_{i}$ chooses between $E_{i}$ and $\bar{E}_{i}$. In any case, once the exponents are fixed a definite orientation on $T P$ is induced from one on $T M$.

We write $d_{i}$ for $\operatorname{dim}_{\mathrm{C}} E_{i}$ and refer to this integer as the dimension of $E_{i}$. In terms of these bundles, the equivariant index theorem of [A-S] now specializes to give the equivariant signature of $M$ in the following form:

$$
\tau(M)=\sum_{\{P\}} \mu(P),
$$

with

$$
\mu(P)=\operatorname{index}\left[d_{S}^{P} \otimes \bigotimes_{i=1}^{k}\left\{\Lambda_{\lambda^{m_{i}}} E_{i} / \Lambda_{-\lambda^{m_{i}}} E_{i}\right\}\right] .
$$

Remarks. In this formula we have used the substitution $e^{i \theta}=\lambda$ as throughout, and $d_{S}^{P}$ denotes the signature operator of $P$, qua oriented manifold.

The twisting bundle of $d_{S}^{P}$ in (8.3) makes good sense in the $K$ theory of $P$, tensored by the rational function in $\lambda$ by virtue of the nilpotence of the ideal, ${ }^{\circ} K(P)$ "of dimension 0 bundles" in $K(P)$. Indeed, given a bundle $E$ of dimension $d$, then in $K(P)$ the class of ${ }^{\circ} E=E-d 1$ has dimension 0 , and the exterior powers of ${ }^{\circ} E$ and $E$ are related by

$$
\Lambda_{a} E=\Lambda_{a}\left({ }^{\circ} E+d \cdot 1\right)=(1+a)^{d} \Lambda_{a /(1+a)}\left({ }^{\circ} E\right) .
$$

Hence the denominator in $(8.3)$ is of the form

$$
\prod\left(1-\lambda^{m_{i}}\right)^{d_{i}}\{1+N\}
$$

where $N$ is a finite linear combination of nilpotent elements in $K(P)$ with coefficients which are monomials in $\lambda^{m_{i}} /\left(1-\lambda^{m_{i}}\right)$; the denominator can therefore be inverted so that the twisting factor $\bigotimes_{i=1}^{k} \Lambda_{\lambda^{m_{i}}} E_{i} / \Lambda_{-\lambda^{m_{i}}} E_{i}$ finally takes on the form of a finite sum

$$
\sum_{(j)} a_{j}^{P}(\lambda) N_{j}
$$

where the $N_{j} \in K(P)$ and the $a_{j}^{P}(\lambda)$ are rational functions whose poles are entirely on the unit circle and possibly at $\infty$ or/and 0 . It follows that

$$
\mu(P)=\sum a_{j}^{P}(\lambda) \cdot \operatorname{index}\left(d_{S}^{P} \otimes N_{j}\right)
$$


is also a rational function in $\lambda$ with this polar behavior. (Actually there are no poles at 0 or $\infty$ in $\mu(P)$ because $\Lambda_{\lambda^{m_{i}}}\left(E_{i}\right) / \Lambda_{-\lambda^{m_{i}}}\left(E_{i}\right)$ at most changes sign under the substitution $\lambda \rightarrow \lambda^{-1}$.)

Hence the rigidity proof for $\tau(M)$ goes forward in the general case just as it did before.

It is easiest to see the genesis from (3.5) to (8.3) if we assume that all of the $E_{i}$ are line bundles because then the twisting factor of $(8.3)$ is simply

$$
\prod\left(1+\lambda^{m_{i}} E_{i}\right) / \prod\left(1-\lambda^{m_{i}} E_{i}\right) \text {, }
$$

so that, when $P$ is simply a point $p$ and consequently $E_{i}=1$ in $K(p t)$, this factor is precisely $\mu(p)$. In this extreme case index $d_{S}^{p}$ is interpreted as the \pm identity map, the sign depending on the induced orientation of $p$.

Using this same technique, now we can easily compute the contribution of $P$ to $\tau_{q}(M)$. The formula is given by

$$
\mu(P)=\tau_{q}\left(P ; \bigotimes \varphi_{\lambda^{m_{i}}}\left(E_{i}\right)\right),
$$

and we will now spell its meaning out in greater detail and explore its nature as a meromorphic function on $T_{q^{2}}$.

First the terms $\varphi_{\lambda^{m_{i}}}\left(E_{i}\right)$. These are well-defined elements in $K(P) \otimes_{\mathbf{Z}} \mathscr{M}\left(T_{q^{2}}\right)$ where $\mathscr{M}\left(T_{q^{2}}\right)$ denotes the ring of meromorphic functions on $T_{q^{2}}$, which we will think of as a meromorphic function on $\mathbf{C}-\{0\}$ invariant under multiplication by $q^{2}$.

Note that in $\S 6$ we already defined $\varphi_{a}(F)$ as an element in $K(M) \otimes_{\mathbf{Z}} \mathbf{C}$ when $|q|<|a|<1$. Thus $\varphi_{\lambda^{m}}(E)$ makes sense for $|q|^{1 / m}<|\lambda|<1$ by the previous definition, but now we want to emphasize that it extends to a well-defined element of $K(P) \otimes_{\mathbf{Z}} \mathscr{M}\left(T_{q^{2}}\right)$, and in particular we want to study its polar structure in $\lambda$.

For this purpose observe that if $E$ is a line bundle over $P$, then we can define $\log E \in{ }^{\circ} K(P) \otimes_{\mathbf{Z}} \mathbf{Q}$ by the formula

$$
\log E=\log \left(1+{ }^{\circ} E\right)=-\sum_{k=1}^{\infty} \frac{{ }^{\circ} E^{k}}{k}(-1)^{k}
$$

because this sum terminates.

Next consider the substitution $\lambda=e^{z}$ so that $\varphi(\lambda)=\widehat{\varphi}(z)$ and define $\varphi^{(k)}(\lambda)$ to be the $k$ th derivative of $\widehat{\varphi}(z)$ relative to $z$ :

$$
\varphi^{(k)}(\lambda) \equiv\left(\frac{d}{d z}\right)^{k} \widehat{\varphi}(z)
$$

These are new meromorphic functions on $T_{q^{2}}$ with degree $k$ poles at 1 and $q$.

With all this understood define

$$
\varphi_{\lambda}(E)=\widehat{\varphi}(z+\log E) \equiv \sum_{0}^{\infty} \frac{(\log E)^{k}}{k !} \cdot \varphi^{(k)}(\lambda) .
$$


Because $\log E$ is nilpotent in $K(P)$ this expression now clearly exhibits $\varphi_{\lambda}(E)$ as an element in $K(P) \otimes_{\mathbf{Z}} \mathscr{M}\left(T_{q^{2}}\right)$.

Now if $E=\bigoplus_{i} E_{i}$ is a sum of line bundles, then $\varphi_{\lambda}(E)=\bigotimes_{i} \varphi_{\lambda}\left(E_{i}\right)$ and so (8.12) defines $\varphi_{\lambda}(E) \in K(P) \otimes \mathscr{M}\left(T_{q^{2}}\right)$ for sums of line bundles, and hence, by the splitting principle, for any complex $E$.

The operation $\psi_{m}$ applied to $\varphi_{\lambda}(E)$ finally produces $\varphi_{\lambda^{m}}(E)$ and this explains the twisting part of the formula (8.9). It remains to recall that just as in $\S 3, \tau_{q}(P ;())$ is shorthand for

$$
\mathrm{ch} \cdot \operatorname{index}\left\{d_{S}^{P} \otimes R_{q}(T) \otimes()\right\} .
$$

Note also that again using the nilpotence of ${ }^{\circ} K(P)$ as in our treatment of $\varphi_{\lambda}(E), R_{q}(T)$ is seen to be a finite expression of the form $\sum a_{r}(q) W_{r}$ in $K(P) \otimes_{\mathbf{Z}} \mathbf{C}$ where the $a_{r}(q)$ are given by convergent power series in $q$ for $|q|<1$.

All in all, then, we see that the contribution of $P$ to $\tau_{q}(M)$ is given by a polynomial in $\psi_{m_{i}} \varphi^{(k)}(\lambda)$ with coefficients that are analytic functions of $q$ in $|q|<1$. As in the case of isolated fixed points, we have identified $\tau_{q}(M)$ as a meromorphic function on $T_{q^{2}}$ and our previous argument may be used to show that this meromorphic function is regular on the circle $|\lambda|=1$. Again, the proof of our rigidity theorem boils down to proving that $\tau_{q}(M)$ has no poles at all.

Now, a priori, poles can appear only at roots of $q^{2}$, that is, on circles of radius $|a|$ where $a=\alpha^{s}$ for $\alpha$ a $k$ th root of $q$ and for $s$ relatively prime to $k$. Also we will rule out such poles by proving that the transfer formulae of (7.5) and (7.12) for $t_{\alpha^{s}} \tau_{q}(M)$ generalize to $S^{1}$ actions without constraints. In fact, the transfer formulae generalize essentially unchanged. If we let $\left[\frac{k}{2}\right]$ denote the greatest integer less than or equal to $\frac{k}{2}$, then, when $M$ is a spin manifold, we still have

$$
t_{\alpha^{s}} \tau_{q}(M)=\tau_{q}\left(M_{k}^{\prime} ; \otimes_{r=1}^{[k / 2]} \varphi_{\alpha^{\omega(r)}}\left(T_{r}\right)\right)
$$

where $\omega(r) \in\{1, \ldots, k-1\}$ is the $\bmod k$ reduction of $s \cdot r$, and where $M_{k}^{\prime} \subseteq M$ is the fixed point manifold of $\mathbf{Z}_{k} \subset S^{1}$ with a specific orientation which we will now describe.

When $k$ is odd, the tangent bundle $\left.T M\right|_{M_{k}}$ decomposes according to (7.4) and induces an orientation on $T M_{k}$. Call this orientation +1 . Let $P$ be a component of the fixed point set of the $S^{1}$ action with $P \subseteq M_{k}$ and choose the signs of the exponents along $P$ so that each $m_{j} \not \equiv 0(\bmod k)$ is congruent $\bmod k$ to some $r \in\{1, \cdots,(k-1) / 2\}$. Choose the orientation of $T P$ and choose the signs of those $m_{j} \equiv 0(\bmod k)$ so that the induced orientation on $\left.T M\right|_{P}$ is the correct one; then, the induced orientation on $\left.T M_{k}\right|_{P}$ will be the +1 orientation. For each $m_{j}$, introduce $\left(\ell_{j}, \omega_{j}\right) \in \mathbf{Z} \times\{1, \ldots, k-1\}$ by

$$
s \cdot m_{j}=\ell_{j} \cdot k+\omega_{j}
$$


and then define

$$
\varepsilon(P)=\sum_{j} d_{j} \cdot \ell_{j}
$$

where $d_{j}=\operatorname{dim}_{\mathrm{C}} E_{j}$.

The orientation for $M_{k}^{\prime}$ is now defined to be $+1 \cdot(-1)^{\varepsilon(P)}$ on that component of $M_{k}$ which contains $P_{j}$, and Lemma 8.1 below insures that $(-1)^{\varepsilon(P)}=$ $(-1)^{\varepsilon\left(P^{\prime}\right)}$ when $P$ and $P^{\prime}$ are both fixed point manifolds of $S^{1}$ in the same component of $M_{k}$.

For the case $k$ even, $\left.T\right|_{M_{k}}$ decomposes according to (7.11), and as $\bigoplus_{r=1}^{k / 2-1} T_{r}^{\#}$ is naturally oriented, an orientation is induced on $T M_{k} \oplus T_{k / 2}$. Choose an orientation for $T M_{k}$ and call it +1 ; this induces one on $T_{k / 2}$.

Let $P$ be a component of the fixed point set of $S^{1}$ and choose the signs of the exponents at $P$ by the folowing rule. If $m_{j} \not \equiv 0, \frac{k}{2} \bmod k$, make the choice as before so that $\left(m_{j}\right)_{\bmod (k)} \in\left\{1, \ldots, \frac{k}{2}-1\right\}$. Choose the signs for those $m_{j} \equiv$ $0, \frac{k}{2}(\bmod k)$ and choose the orientation of TP to make the induced orientation on $\left.T M_{k} \oplus T_{k / 2}\right|_{P}$ correct. This will make the induced orientation on $\left.T M\right|_{P}$ correct. However, the induced orientation of $\left.T M_{k}\right|_{P}$ may not be the correct one $(+1)$. To keep track of the discrepancy, introduce $\varepsilon_{0}=0,1$ with $\varepsilon_{0}=0$ if the induced orientation on $\left.T M_{k}\right|_{P}$ is correct, and $\varepsilon_{0}=+1$ if the induced orientation on $\left.T M_{k}\right|_{P}$ is incorrect. (If $\bigoplus_{j: m_{j} \equiv k / 2 \bmod k} E_{j}$ equivariantly splits an odd (complex) dimensional subbundle, it is always possible to choose the signs of the $m_{j} \equiv 0, \frac{k}{2}(\bmod k)$ and the orientation of $P$ to get the correct orientation for both $\left.T M\right|_{P}$ and $\left.T M_{k}\right|_{P}$.)

For each $m_{j}$, introduce $\left(\ell_{j}, \omega_{j}\right)$ by $(8.14)$ and set

$$
\varepsilon(P)=\varepsilon_{0}+\sum_{j} d_{j} \cdot \ell_{j}
$$

The orientation for $M_{k}^{\prime}$ is again $+1 \cdot(-1)^{\varepsilon(P)}$ on that component of $M_{k}$ that contains $P$, and the assertion that this orientation is unambiguous is made in Lemma 8.1. The proof of Lemma 8.1 is deferred to the next section.

Lemma 8.1. Let $M$ be an oriented spin manifold on which $S^{1}$ acts and for $a$ positive integer $k$, let $M_{k} \subseteq M$ be a connected component of the fixed point set of $\mathbf{Z}_{k} \subset S^{1}$. Let $s \in \mathbf{Z}$ be relatively prime to $k$. Let $P, P^{\prime}$ be connected fixed point manifolds for the $S^{1}$-action, both lying in $M_{k}$. Use the prescription above to define the numbers $\varepsilon(P)$ and $\varepsilon\left(P^{\prime}\right)$ in (8.15) for $k$ odd and (8.16) for $k$ even. Then $(-1)^{\varepsilon(P)}=(-1)^{\varepsilon\left(P^{\prime}\right)}$.

To prove our general transfer formula (8.13), we begin with the remark that when we choose our exponents at $P$ according to the preceding prescription, then the analog of (7.10) still holds:

$$
t_{\alpha^{s}} \mu_{P}=(-1)^{\varepsilon(P)} \cdot \tau_{q}\left(P ; \bigotimes \varphi_{\lambda^{m_{i} \alpha^{\left(j_{i}\right.}}}\left(E_{i}\right)\right)
$$


We see (8.17) by using the antiperiodicity $\varphi(\lambda q)=-\varphi(\lambda)$ to first prove

$$
t_{\alpha^{s}} \varphi\left(\lambda^{m}\right)=\varphi\left(\lambda^{m} \alpha^{m s}\right)=(-1)^{\ell} \varphi\left(\lambda^{m} \alpha^{\omega}\right)
$$

for $(\ell, \omega) \in \mathbf{Z} \times\{1, \cdots, k-1\}$ defined by $s \cdot m=\ell \cdot k+\omega$. Now we observe that $(8.17)$ is a direct consequence of $(8.18)$ when we go back to (8.12) to define $\varphi_{a}(E)$.

Now, as before, we observe that $\tau_{q}\left(P \otimes_{i} \varphi_{\lambda^{m_{i} \alpha^{(j i}}}\left(E_{i}\right)\right)$ is the contribution from $P$ to the fixed point expression for $\tau_{q}\left(M_{k} ; \bigotimes_{r=1}^{[k / 2]} \varphi_{\alpha^{\omega(r)}}\left(T_{r}\right)\right)$. The argument is the same as before. Since Lemma 8.1 insures that $(-1)^{\varepsilon(P)}$ depends only on the component of $M_{k}$ that contains $P$, we can define $M_{k}^{\prime}$ as $M_{k}$ with the new orientation and our transfer formula is complete.

\section{EXPONENTS MOD 2}

The proofs of Lemmas 7.1 and 8.1 presented here compare the exponents at different points of $M$ by means of judicious equivariant maps of the standard two sphere $S^{2}$ into $M$. These maps pull back equivariant vector bundles on $M$ to $S^{2}$ where we can exploit more or less standard facts concerning equivariant bundles on $S^{2}$.

Then let $S^{1}$ act on $S^{2}=S_{(1)}^{2}$ by rotations about the north-south axis in the standard manner, and let $S_{(k)}^{2}$ denote the $k$ th iterate of this action, which rotates $S^{2} k$ times about its axis as we go once around $S^{1}$. These two actions of $S^{1}$ on $S^{2}$ are related by an $S^{1}$ equivariant map

$$
\begin{gathered}
S^{2} \\
\pi_{k} \downarrow \\
S_{(k)}^{2}
\end{gathered}
$$

which is of degree $k$, and which, under stereographic projection of $S^{2}$ onto $\mathrm{C} \cup \infty$, corresponds to the map $z \rightarrow z^{k}$. The first of the two facts we need concerns complex bundles over $S^{2}=S_{(1)}^{2}$.

Lemma 9.1. Let $E$ be a complex vector bundle over $S_{(1)}^{2}$ to which the $S^{1}$ action is lifted. Then the first Chern class of $E$ is given by

$$
\int_{S^{2}} c_{1}(E)=\sum\left(m_{i}^{n}-m_{i}^{s}\right)
$$

where the $m_{i}^{n}$ and $m_{i}^{s}$ are the exponents of the $S^{1}$ action on $E$ at the north and south poles, respectively.

The second fact we need is an equivariant generalization of the statement that all even-dimensional real bundles $E^{\#}$ over $S^{2}$ admit complex structures.

Lemma 9.2. Every real, oriented, even dimensional, equivariant bundle $E^{\#}$ over $S_{(k)}^{2}$ is the underlying real oriented bundle of a complex equivariant vector bundle $E$ over $S_{(k)}^{2}$. 
Now Lemma 7.1 is a special case of Lemma 8.1 , which can be generalized by considering, rather than $T M$, any real, oriented vector bundle $V \rightarrow M$ with $w(V)=0$ and to which our $S^{1}$ action lifts. Restricting $V$ to $M_{k}$ produces a vector bundle $\left.V\right|_{M_{k}}$ on which $\mathrm{Z}_{k} \hookrightarrow S^{1}$ acts; just rotate $2 \pi / k$ radians around $S^{1}$. As in the previous section, this $\mathbf{Z}_{k}$ action decomposes $\left.V\right|_{M_{k}}$ into constituents

$$
\left.V\right|_{M_{k}}=V_{0}+V_{1}^{\#}+\cdots+V_{(k-1) / 2}^{\#}
$$

if $k$ is odd, and into constituents

$$
\left.V\right|_{M_{k}}=V_{0}+V_{1}^{\#}+\cdots+V_{k / 2-1}^{\#}+V_{k / 2}
$$

if $k$ is even. Here $\mathbf{Z}_{k}$ acts trivially on $V_{0}$, the generator acts by $(-1)$ on $V_{k / 2}$, and each $V_{r}^{\#}$ for $1 \leq r<\frac{k}{2}$ has a unique complex structure with the property that the generator of $\mathbf{Z}_{k}$ acts on the resulting complex bundle, $V_{r}$, by $\xi \rightarrow \xi^{r}$.

When $k$ is odd, (9.2) orients $V_{0}$. When $k$ is even, (9.3) orients $V_{0} \oplus V_{k / 2}$ and, looking ahead to Lemma 10.1, we assert that $V_{0}$ is orientable. Assuming this, fixing an orientation on $V_{0}$ orients $V_{k / 2}$.

At a component, $P$, of the fixed point set of $S^{1}, V$ decomposes as

$$
V=E_{0} \oplus \bigoplus_{i} E_{i}^{\#}
$$

where $S^{1}$ acts trivially on $E_{0}$ and where each $E_{i}^{\#}$ is the realization of a complex bundle, $E_{i} \rightarrow P$, on which $S^{1}$ acts by $\lambda \rightarrow \lambda^{m_{i}}$ for some $m_{i} \in \mathbf{Z}$. As with $V=T M$, the exponents $\left\{m_{i}\right\}$ are canonical up to sign, as $\left\{E_{i}\right\}$ are canonical up to conjugation and choosing the signs for $\left\{m_{i}\right\}$ specifies $\left\{E_{i}\right\}$. Choose these signs compatibly with orientation by requiring those $m_{i} \not \equiv 0, \frac{k}{2}(\bmod k)$ to have mod $k$ reduction in $\{1, \cdots(k-1) / 2\}$ or $\left\{1, \cdots, \frac{k}{2}-1\right\}$ depending on whether $k$ is odd or even. When $k$ is odd, choose the orientation for $E_{0}$ and the signs of those $m_{i} \equiv 0(\bmod k)$ so that the induced orientation on $\left.V_{0}\right|_{P}=E_{0} \oplus \bigoplus_{i: m_{i} \equiv 0(\bmod k)} E_{i}^{\#}$ is also correct. When $k$ is even, choose the signs of those $m_{i} \equiv 0, \frac{k}{2}(\bmod k)$ and the orientation of $E_{0}$ so that the induced orientation on $\left.V_{0} \oplus V_{k / 2}\right|_{P}$ is correct.

It may be impossible to choose the signs of those $m_{i} \equiv 0, \frac{k}{2}(\bmod k)$ and then the orientation of $E_{0}$ to obtain correct orientations for both $\left.V_{0} \oplus V_{k / 2}\right|_{P}$ and $\left.V_{0}\right|_{P}$. Let $\varepsilon_{0}=0$ when the correct orientation for $\left.V_{0}\right|_{P}$ is obtained (one can always arrange this when $\bigoplus_{i: m_{i}=k / 2(\bmod k)} E_{i}$ splits equivariantly with an odd complex dimensional summand) and let $\varepsilon_{0}=+1$ when $\left.V_{0}\right|_{P}$ inherits the wrong orientation.

For any $s$ relatively prime to $k$, introduce $\left\{\left(\ell_{i}, \omega_{i}\right)\right\} \subset \mathbf{Z} \times\{1, \ldots, k-1\}$ by $s \cdot m_{i}=\ell_{i} \cdot k+\omega_{i}$; and then introduce $\varepsilon(P)=\varepsilon_{0}+\sum_{i} d_{i} \cdot \ell_{i}$ where $d_{i}=\operatorname{dim}_{\mathrm{C}} E_{i}$.

Now we can state our generalization of Lemma 8.1. 


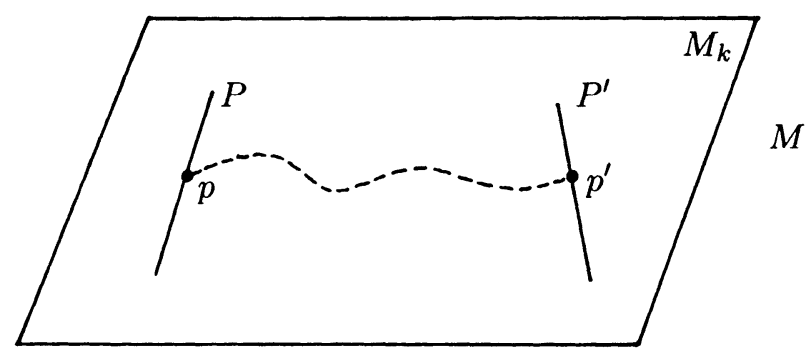

FIGURE 3

Lemma 9.3. Let $M$ be a smooth manifold and let $V \rightarrow M$ be a real, oriented vector bundle with $w_{2}(V)=0$. Suppose $S^{1}$ acts on $M$ and compatibly on $V$ and, for $k \in \mathbf{Z}$, let $M_{k} \subseteq M$ be a connected component of the set in $M$ fixed under $\mathbf{Z}_{k} \subset S^{1}$. Let $P, P^{\prime} \subset M_{k}$ be distinct, connected components of the set in $M_{k}$ fixed under $S^{1}$ and define the numbers $\varepsilon(P), \varepsilon\left(P^{\prime}\right)$ by the prescription in the preceding paragraphs. Then $\varepsilon(P)-\varepsilon\left(P^{\prime}\right)$ is an even number.

We will now turn to the proof of Lemma 9.1. Choose a point $p \in P$ and a point $p^{\prime} \in P^{\prime}$. The situation is depicted in Figure 3, where the dotted line represents a path from $p$ to $p^{\prime}$ which lies in $M_{k}$ and is so constructed that it avoids all other fixed points of our action, and with the property that rotating the path via our $S^{1}$ action induces an equivariant map of $S^{2}$ into $M_{k}$,

$$
f: S^{2} \rightarrow M_{k} \text {, }
$$

taking the north pole to $p$ and the south pole to $p^{\prime}$.

Since the path in Figure 3 lies in $M_{k}$, a second map from $S^{2}$ into $M_{k}$ is constructed by rotating the path through an angle of $2 \pi / k$ radians by our $S^{1}$ action.

This new map is denoted by

$$
f_{k}: S^{2} \rightarrow M_{k}
$$

and in the equivariant context, $f_{k}$ fits into the commutative diagram

$$
\begin{gathered}
S^{2} \downarrow \\
\pi_{(k)}^{2}
\end{gathered} \underset{f_{k}}{\longrightarrow} M_{(k)}
$$

We now pull the bundles $V_{r}$ back to $S_{(k)}^{2}$ via $f_{k}$. For $r \neq 0, \frac{k}{2}$ these $f_{k}^{*} V_{r}$ are complex equivariant bundles a priori, and for $r=0$ or $\frac{k}{2}$, Lemma 9.2 allows us to think of $f_{k}^{*} V_{r}$ as a complex bundle also.

In all cases, $f^{*} V_{r}=\left(\pi_{k} \circ f_{k}\right)^{*} V_{r}$ is an $S^{1}$-equivariant, complex bundle for the standard $S^{1}$ action on $S^{2}$ and restricts equivariantly to $n$ (=north pole) as a direct sum of lines $f^{*} V_{r} l_{n}=\bigoplus_{\alpha} L_{\alpha}(r)$, and $S^{1}$ acts on $L_{\alpha}(r)$ by 
$\lambda \rightarrow \lambda^{m_{n}(r)}$. For $r \neq 0, \frac{k}{2}(\bmod k)$, the set of exponents $\left\{m_{\alpha}(r)\right\}$ is precisely the subset $\left\{m_{i}: m_{i} \equiv r(\bmod k)\right\}$ of the set of exponents for the action of $S^{1}$ on $\left.V\right|_{P}$, but counted with multiplicity $d_{i}=\operatorname{dim}_{\mathrm{C}} E_{i}$. For $k$ odd and $r=0,\left.f^{*} V_{0}\right|_{n}$ also decomposes as a sum $\bigoplus_{\alpha} L_{\alpha}(0)$ of complex lines on which the $S^{1}$ action is defined by exponents $\left\{m_{\alpha}(0)\right\}$ and this is precisely the subset $\left\{m_{i}: m_{i} \equiv 0(\bmod k)\right\}$ after counting with multiplicity $d_{i}$. When $k$ is even, either $\varepsilon_{0}=0$ or 1 and if $\varepsilon_{0}=0$, then one has the same situation for $\left.f^{*} V_{0}\right|_{n}$ and a similar situation for $f^{*} V_{k / 2} l_{n}$ which defines exponents $\left\{m_{\alpha}\left(\frac{k}{2}\right)\right\}_{\alpha=1}^{\operatorname{dim}_{\mathrm{C}} V_{k / 2}}$ as the subset $\left\{m_{i}: m_{i} \equiv \frac{k}{2}(\bmod k)\right\}$ after counting with multiplicity $d_{i}$. However, when $\varepsilon_{0}=+1$, the orientations on $\left.f^{*} V_{0}\right|_{n}$ and $\left.f^{*} V_{k / 2}\right|_{n}$ must be corrected so the exponents for the line decomposition of $\left.f^{*} V_{0}\right|_{n}$ are $\left\{m_{\alpha}(0)\right\}$ which differs from the subset $\left\{m_{i}: m_{i} \equiv 0(\bmod k)\right\}$ counted with multiplicity in that one $m_{\alpha}(0)$ has the wrong sign. Likewise, the exponents for $\left.f^{*} V_{k / 2}\right|_{n}$ are a set $\left\{m_{\alpha}\left(\frac{k}{2}\right)\right\}_{\alpha=1}^{\operatorname{dim}_{\mathrm{C}} V_{k / 2}}$ which differs from the set $\left\{m_{i}: m_{i} \equiv \frac{k}{2}(\bmod k)\right\}$ counted with multiplicity in that one $m_{\alpha}\left(\frac{k}{2}\right)$ has the wrong sign. (Changing an odd number of complex lines to the conjugate line will switch the orientation.)

We likewise introduce the set of exponents $\left\{m_{\alpha}^{\prime}(r)\right\}$ for the $S^{1}$-action on the restriction to the south pole of $f^{*} V_{r}$.

With all of this understood, Lemma 9.1 tells us that

$$
\sum_{\alpha}\left(m_{\alpha}(r)-m_{\alpha}^{\prime}(r)\right)=\int_{S^{2}} c_{1}\left(f^{*} V_{r}\right)
$$

and since $\pi_{k}: S^{2} \rightarrow S_{(k)}^{2}$ is a degree $k$ map, we can conclude that

$$
\sum_{\alpha}\left(m_{\alpha}(r)-m_{\alpha}^{\prime}(r)\right)=k \cdot \int_{S^{2}} c_{1}\left(f_{k}^{*} V_{r}\right) .
$$

Next, let $s$ be relatively prime to $k$. Since $m_{\alpha}(r)$ is an exponent of $V_{r}$ at $p, r$ equals its mod $k$ reduction, and likewise for $m_{\alpha}^{\prime}(r)$. Thus $s \cdot m_{\alpha}(r)$ and $s \cdot m_{\alpha}^{\prime}(r)$ all have the same $\bmod k$ reduction, $(s \cdot r)_{\bmod k}$ which we fix once and for all. Hence, integers $\left\{\ell_{\alpha}(r)\right\}$ are defined at $p$ by

$$
s \cdot m_{\alpha}(r)=\ell_{\alpha}(r) \cdot k+(s \cdot r)_{\bmod k} .
$$

Similarly we obtain $\left\{\ell_{\alpha}^{\prime}(r)\right\}$. Hence, if we divide (9.8) by $k$ and multiply by $s$ we find that

$$
\sum_{\alpha}\left(\ell_{\alpha}(r)-\ell_{\alpha}^{\prime}(r)\right)=s \cdot \int_{S^{2}} c_{1}\left(f_{k}^{*}\left(V_{r}\right)\right) .
$$

Now if $r \not \equiv 0, \frac{k}{2}(\bmod k)$, then

$$
\sum_{\alpha} \ell_{\alpha}(r)=\sum_{i: m_{i} \equiv r(\bmod ) k} d_{i} \cdot \ell_{i}
$$

since each $m_{i} \equiv r(\bmod k)$ appears in the set $\left\{m_{\alpha}(r)\right\}$ with multiplicity $d_{i}$. If $k$ is odd, (9.12) holds for $r=0$ also, and if $k$ is even, (9.12) holds for 
$r=0$ and $\frac{k}{2}$ provided that $\varepsilon_{0}=0$. However, if $\varepsilon_{0}=+1$, then one $m_{\alpha}(0)$ and one $m_{\alpha}\left(\frac{k}{2}\right)$ have the "wrong" signs and so

$$
\sum_{\alpha} \ell_{\alpha}(0)=\left(\sum_{i: m_{i} \neq 0(\bmod k)} d_{i} \cdot \ell_{i}\right)-2 \cdot \varepsilon_{0} \cdot \ell_{1}(0)
$$

and

$$
\sum_{\alpha} \ell_{\alpha}\left(\frac{k}{2}\right)=\left(\sum_{i: m_{i} \equiv k / 2(\bmod k)} d_{i} \cdot \ell_{i}\right)-2 \cdot \varepsilon_{0} \cdot \ell_{1}\left(\frac{k}{2}\right)-\varepsilon_{0}
$$

give correct formulae for $\varepsilon_{0}=0$ or 1 .

Now similar formulae hold at the south pole, so summing over $r$, we obtain

$$
\sum_{i}\left(d_{i} \ell_{i}-d_{i} \ell_{i}^{\prime}\right)-\varepsilon_{0}+\varepsilon_{0}^{\prime}-2 \varepsilon_{0}\left[\ell_{1}(0)+\ell_{1}\left(\frac{k}{2}\right)-\ell_{1}^{\prime}(0)-\ell_{1}^{\prime}\left(\frac{k}{2}\right)\right]=s \cdot \int_{S^{2}} c_{1}\left(f_{k}^{*}\left(V_{r}\right)\right) .
$$

But this establishes our lemma because the spin hypothesis on $V$ implies that $\mathrm{w}_{2}(V)=0$, whence $\mathrm{w}_{2}\left(f_{k}^{*} V\right)=0$ so that $c_{1}\left(f_{k}^{*} V\right)$ is even! But then $\sum\left(\ell_{i}-\ell_{i}^{\prime}\right)+\varepsilon_{0}-\varepsilon_{0}^{\prime}$ is even, as was to be shown. QED

For the sake of completeness, we now give proofs of Lemmas 9.1 and 9.2.

Proof of Lemma 9.1. Since $S^{1}$ acts on $E$ we can construct the vector bundle

$$
\begin{array}{ll}
E_{S_{1}} & =E \times \times_{S^{1}} E S_{1} \\
\downarrow & \downarrow \\
S_{S^{1}}^{2} & =S^{2} \times_{S^{1}} E S^{1}
\end{array}
$$

where $E S^{1}$ is the universal $S^{1}$-bundle over the classifying space $B S^{1}$. As a complex vector bundle, it has a first Chern class, $c_{1}\left(E_{S^{1}}\right) \in H^{2}\left(S^{2} \times_{S^{1}} E S^{1}\right)$. Now the projection $\pi: E S^{1} \rightarrow B S^{1}$ identifies $S^{2} \times_{S^{1}} E S^{1}$ as a fiber bundle over $B S^{1}$ with fiber $S^{2}$. Two canonical sections, $N$ and $S$, correspond to the north and south poles of $S^{2}$, respectively.

A typical fiber $F$ of this bundle defines a class $[F] \in H_{2}\left(S^{2} \times_{S^{1}} E S^{1}\right)$. Two additional classes are defined by $N_{*}\left[\mathbf{P}^{1}\right]$ and $S_{*}\left[\mathbf{P}^{1}\right]$ where $\left[\mathbf{P}^{1}\right] \in H_{2}\left(B S^{1}\right)$ is the generator. An easy exercise shows that

$$
N_{*}\left[\mathbf{P}^{\mathrm{l}}\right]-S_{*}\left[\mathbf{P}^{1}\right]=[F] \text {, }
$$

and our formula in Lemma 9.1 is obtained by evaluating $c_{1}\left(E_{S^{1}}\right)$ on both sides of (9.16).

Proof of Lemma 9.2. Rotating $S^{1}$ through $2 \pi / k$ radians gives the identity on $S_{(k)}^{2}$ and so generates a $\mathbf{Z}_{k}$ action on $E^{\#}$ which decomposes $E^{\#}$ in the usual way, i.e., as

$$
E^{\#}=E_{0}+E_{1}^{\#}+\cdots+E_{(h-1) / 2}^{\#}
$$


or as

$$
E^{\#}=E_{0}^{\#}+E_{1}^{\#}+\cdots+E_{h / 2-1}^{\#}+E_{h / 2}^{\#}
$$

depending on whether $k$ is odd or even. For $r \neq 0, \frac{k}{2}$ each $E_{r}^{\#}$ has a complexification to $E_{r}$ on which the $\mathrm{Z}_{k}$ generator acts as $\xi^{r}$, so we need only concern ourselves with $r=0, \frac{k}{2}$. First, we observe

Lemma 9.4. Let $E^{\#}$ be as in Lemma 9.2 with $k$ even. Decompose $E^{\#}$ as in (9.18); then $E_{0}^{\#}$ and $E_{k / 2}^{\#}$ are even dimensional.

Proof of Lemma 9.4. As $E^{\#}$ is even dimensional, we may consider $E^{\#}=$ $E_{0}^{\#} \oplus E_{k / 2}^{\#}$. As $\mathbf{Z}_{k} \subset S^{1}$, it acts as an orientation preserving automorphism of $E^{\#}$, hence on the real line bundle $\operatorname{det} E^{\#}=\operatorname{det} E_{0}^{\#} \otimes \operatorname{det} E_{k / 2}^{\#}$ it must act as +1 . But it acts a priori as +1 on $\operatorname{det} E_{0}^{\#}$ and it acts as $(-1)^{\operatorname{dim} E_{k / 2}^{\#}}$ on $\operatorname{det} E_{k / 2}^{\#}$ so $\operatorname{dim} E_{k / 2}^{\#}$ is even. QED

Now our standard $S^{1}$ action (rotation once about $S^{1}$ gives one rotation of $S^{2}$ ) lifts to $E_{0}^{\#}$. At the north and south poles we may give $E_{0}^{\#}$ a complex structure which is compatible with the linear $S^{1}$ action on the fiber and with the orientation. Indeed, we can choose our exponents $\left(m_{1}, \ldots, m_{\left(\operatorname{dim} E_{0}^{*}\right) / 2}\right)$ which are $0(\bmod k)$ at $n$ and $s$ to uniquely define such a complex structure. Now pick a longitude, $\ell$, and extend our complex structures at $n$ and $s$ to a complex structure for $E_{0}^{\#} \mid \ell$. We can do this because $S O(2 n) / U(n)$ is path connected. Since each $S^{1}$ orbit save $n$ and $s$ intersects $\ell$ exactly once, we can use the $S^{1}$ action to extend the complex structure along $\ell$ to a global one which is, a priori, $S^{1}$-equivariant.

For $E_{k / 2}^{\#}$ we can make the same construction. Though it is true here that rotating once around $S^{2}$ does not lift to the identity on $E_{k / 2}^{\#}$, rather it lifts to $(-1)$. However, $(-1) \in S O(2 n)$ lies in $U(n)$, so it does not hinder our construction.

\section{Orientation}

It was essential for our program that the fixed point set $M_{n} \subset M$ of $\mathbf{Z}_{n} \subset S^{1}$ be orientable, provided $M$ is spin. This result is a special case of a more general phenomenon which arises in the context of a $\mathrm{Z}_{n}$ action on a manifold $M$, and a real, oriented vector bundle, $V \rightarrow M$, to which the $\mathrm{Z}_{n}$ action lifts. When restricted to the fixed point manifold, $M_{n} \subset M$ for $\mathrm{Z}_{n},\left.V\right|_{M_{n}}$ admits a $\mathbf{Z}_{n}$ action which decomposes $\left.V\right|_{M_{n}}$ in the usual way:

$$
\left.V\right|_{M_{n}}=V_{0}+V_{1}^{\#}+\cdots+V_{(n-1) / 2}^{\#}
$$

if $n$ is odd, or

$$
\left.V\right|_{M_{n}}=V_{0}+V_{1}^{\#}+\cdots+V_{n / 2-1}^{\#}+V_{n / 2}
$$


if $n$ is even, where the generator of $\mathrm{Z}_{n}$ acts trivially on $V_{0}$, by $(-1)$ on $V_{n / 2}$ and where $V_{r}^{\#}$ for $1 \leq r<n / 2$ have natural complex structures.

Lemma 10.1. If $V$ is spin and if the $\mathbf{Z}_{n}$ action preserves the orientation and the spin structure of $V$, then $V_{0}$ is orientable.

Taking $V=T M$ produces our result that $M_{n} \subset M$ is orientable for $M$ a spin manifold. As we remarked, this corollary can be deduced from a theorem of Edmonds [E]; his theorem asserts that the fixed point set of an involution on a spin manifold is orientable.

However, we will present a proof of this lemma along different lines from his. To begin, we note that for $n$ odd, $V$ needs only to be orientable for $V_{0}$ to be orientable, as the other constituents of $V$ have complex structures.

For the $n$ even case, we base our proof on the following quite general proposition.

Proposition 10.2 (Bott-Samelson [B-S]). In a connected and simply-connected compact Lie group the centralizer of an element is connected.

Gist of proof. Let $G$ be the group and $g$ the element and consider the orbit of $g$ under the adjoint action of $G$ on itself. We write $O_{g}$ for this orbit, and clearly $O_{g} \simeq G / C_{g}$, where $C_{g}$ is the centralizer of $G$. Under our assumptions it follows from the homotopy exact sequence that

$$
\pi_{0}\left(C_{g}\right) \simeq \pi_{i}\left(O_{g}\right)
$$

Now let $\Omega\left(G ; O_{g}\right)$ be the space of paths on $G$ ending on $O_{g}$. It has the same homotopy type as $O_{g}$. Projection on the initial point of such a path gives us a fibering

$$
\Omega_{e}\left(G, O_{g}\right) \longrightarrow \Omega\left(G ; O_{g}\right) \longrightarrow G,
$$

with fiber the space of paths in $G$ starting at $e$ and ending on $O_{g}$. The space on the left is simply connected by an old theorem of Bott and Samelson [B-S]. In fact, by Morse theory $\Omega_{e}\left(G ; O_{g}\right)$ is torsion free and has only even-dimensional homology.

With the aid of this result we can prove Lemma 10.1 in the following manner. Let $Q$ be the principal Spin $(d)$ bundle covering the oriented frame bundle $P$ of $V$ and let $f: M \rightarrow M$ be the map induced by the action of $e^{2 \pi i / n} \in \mathbf{Z}_{n}$ on $M$. Then $M_{n}=M^{f}$ is the fixed point set of $f$ and by assumption we have induced bundle maps $f_{Q}: Q \rightarrow Q$ and $f_{P}: P \rightarrow P$ covering $f$.

Let $Q^{\prime}$ be the preimage of a connected component of $M^{f}$ in $Q$. Then $f_{Q} \mid Q^{\prime}$ covers the identity automorphism of $Q^{\prime}$. Therefore, $f_{q} \mid Q^{\prime}$ is induced by an equivariant map $h: Q^{\prime} \rightarrow \operatorname{Spin}(n)$ in the sense that

$$
f_{Q}(q)=q \cdot h(q), \quad q \in Q^{\prime} .
$$

Equivariance implies that $f_{Q}(q \cdot g)=f_{Q}(q) \cdot g$ which translates to

$$
h(g \cdot g)=g^{-1} h(q) g \text {. }
$$


This $h$ maps the fibers of $Q^{\prime}$ to Ad-orbits of $\operatorname{Spin}(d)$. Now $f_{Q}$ being of finite order, $f_{Q} \mid Q^{\prime}$ is also, so that $h$ takes values in orbits of points of finite order. These being discrete, we see that $h$ actually takes values in a fixed orbit $O_{g}$ :

$$
h: Q \longrightarrow O_{g}
$$

Let $g \in O_{g}$ be a point of this orbit and consider the inverse image

$$
Q^{\prime \prime}=h^{-1}(g) \text {. }
$$

Then it is clear that $Q^{\prime \prime} \subset Q^{\prime}$ has $C_{g}$ as its structure group. In short, along $M^{f}$ the structure group of $Q$ is naturally broken to $C_{g}$.

Consider now the projection of $Q^{\prime \prime}$ to the oriented orthogonal frame bundle $P$ of $V$. The structure group of $P$ is $S O(n)$, and along $M^{f}$ it is broken to the centralizer $\underline{C}$ of an element $\underline{g}$ in $S O(n)$, which might well have $t w o$ components. (Indeed an involution breaks $O(n)$ into $O(n-k) \times O(k)$ and hence $S O(n)$ to a two-component subgroup of $O(n-k) \times O(k)$.) These two components of the reduction along each fiber of $P \mid M^{f}$ define a double cover $\widetilde{M}^{f}$ of $M^{f}$ and the orientability of $V_{0}$ clearly hinges on the question of whether this double cover is trivial or not.

Now, then, we see from the connectedness of $C_{g}$ that the projection of $Q^{\prime \prime}$ into $P \mid M^{f}$ singles out one component of this double cover, whence $\widetilde{M}^{f}=$ $M^{f} \times \mathbf{Z}_{2}$. QED

\section{Generalizations}

Witten considered a generalization of the basic rigidity theorem by introducing an auxiliary $\operatorname{Spin}(2 k)$ principal bundle $Q_{V}$ over our spin manifold $M$ to which the double of the $S^{1}$ action lifts. Let $\Delta(V)$ and $V$ denote the vector bundles over $M$ which are associated to $Q_{V}$ via the spinor and vector representations, respectively. Similarly we write $\Delta(T)$ to denote the bundle of spinors which is associated to a choice of spin structure on our spin manifold $M$ and in terms of these we introduce the expression

$$
E_{q}(V / T)=\left(\Delta(V) \bigotimes_{n=1}^{\infty} \Lambda_{q^{n}} V\right) \cdot\left(\Delta(T) \bigotimes_{m=1}^{\infty} \Lambda_{q^{m}} T\right)^{-1}
$$

as a formal power series in $q$ with coefficients in the real $K$-theory of $M$. Witten conjectured that

$$
\tau_{q}(M ; E) \equiv \operatorname{ch} \cdot \operatorname{ind}\left(d_{S} \otimes R_{q}(T) \otimes E_{q}(V / T)\right)
$$

is rigid when the equivariant characteristic classes of $V$ are suitably constrained.

To explain these constraints recall that the "equivariant cohomology" of an $S^{1}$-manifold $M$ is defined as the cohomology of an auxiliary space $M_{S}$, constructed as follows. Let $E S$ be a contractible $S^{1}$-space, e.g., the unit sphere in 
a Hilbert space of infinite dimensions on which $S^{1}$ acts by complex multiplication.

Then define $M_{S}$ by

$$
M_{S}=M \times E S / S^{1}
$$

where the quotient is taken relative to the product action on $M \times E S$.

This space fibers over $E S / S^{1}=B S^{1}$, which in this case is just $\mathbf{C} P_{\infty}$. Thus

$$
H_{S}^{*}(p t) \equiv H^{*}\left(\mathbf{C} P_{\infty}\right)=\mathbf{Z}[u], \quad u \in H_{S^{1}}^{2},
$$

so that $H_{S}^{*}(M) \equiv H^{*}\left(M_{S}\right)$ is naturally a module over this ring. Equivariant maps $f: M \rightarrow M^{\prime}$ induce maps $f_{S}: M_{S} \rightarrow M_{S}^{\prime}$ and induce $f_{S}^{*}: H_{S}^{*}\left(M^{\prime}\right) \rightarrow$ $H_{S}^{*}(M)$, in short, $H_{S}^{*}$ is a generalized cohomology theory over $\mathbf{Z}[u]$.

Similarly $S^{1}$-bundles $V$ over $M$ extend to bundles $V_{S}$ over $M_{S}$

$$
V_{S}=V \times E S / S^{1}
$$

so that their characteristic classes are naturally defined in $H_{S}^{*}(M)$. Thus $\mathrm{w}_{2}\left(V_{S}\right)$ and $p_{1}\left(V_{S}\right)$ are well defined in $H_{S}^{*}\left(M ; \mathbf{Z}_{2}\right)$ and $H_{S}^{*}(M ; \mathbf{Z})$, respectively.

The class $\mathrm{w}_{2}\left(V_{S}\right) \in H_{S}^{2}\left(M ; \mathbf{Z}_{2}\right)$ plays the role of an obstruction to an equivariant spin structure on $V$, and it is a fact, which we learned from Dan Freed, that if $\mathrm{w}_{2}(E)=0$ for a real, oriented bundle $E$ over a space $X$, then there exists a canonical class $e(E) \in H^{4}(X ; \mathbf{Z})$ with

$$
p_{1}(E)=2 \cdot e(E) \text {. }
$$

We write $\frac{1}{2} p_{1}(E)$ for this class, as is the tradition among physicists.

In any case the pertinent conditions which Witten proposed for the rigidity of (11.2) are now expressed by

$$
\mathrm{w}_{2}\left(V_{S}-T_{S}\right)=0, \quad \frac{1}{2} p_{1}\left(V_{S}-T_{S}\right)=0
$$

where these classes are taken in $H_{S}^{*}(M)$. Actually, our proof of his conjectures works under the following slightly weaker hypotheses. For each $k>1$, consider $\mathbf{Z}_{k} \subset S^{1}$, the cyclic subgroup of order $k$.

We have the $\mathbf{Z}_{k}$ equivariant cohomology of $M$, defined by

$$
H_{\mathbf{Z}_{k}}^{*}(M)=H^{*}\left(M \times E S / \mathbf{Z}_{k}\right)
$$

and there is a natural "forgetful" map

$$
\alpha\left(S^{1} ; \mathbf{Z}_{k}\right): M \times E S^{1} / \mathbf{Z}_{k} \rightarrow M_{S}
$$

which induces by pullback

$$
\alpha\left(S^{1}, \mathbf{Z}_{k}\right)^{*}: H_{S}^{*} \rightarrow H_{\mathbf{Z}_{k}}^{*}
$$

The arrow which forgets the $S^{1}$ action altogether we denote by $\alpha\left(S^{1} ; 1\right)$. Thus

$$
\alpha\left(S^{1}, 1\right)^{*}: H_{S}^{*} \rightarrow H^{*}
$$


is induced by the inclusion of $M$ into $M_{S}$ as a fiber over $B S$.

Finally, note that if $\mathbf{Z}_{k}$ acts trivially on a space $X$ then there is a new arrow

$$
\pi^{*}: H^{*}(X) \rightarrow H_{\mathbf{Z}_{k}}^{*}(X)
$$

induced by the projection

$$
X_{\mathbf{Z}_{k}}=\left(X \times E S^{1}\right) / \mathbf{Z}_{k}=X \times B \mathbf{Z}_{k} \stackrel{\pi}{\rightarrow} X .
$$

With these concepts understood, we let $\mathrm{Z}_{\infty} \equiv S^{1}$ and then we have the following proposition.

Proposition 11.1. Let $M$ be compact oriented and spin, and let $V$ be an auxiliary spin bundle over $M$. For each $1<k \leq \infty$, let $i: M_{k} \rightarrow M$ be the inclusion of the fixed point set of $\mathbf{Z}^{k} \subset S^{1}$ in $M$ and so $i$ induces $i_{S}: M_{k} \times_{S^{1}} E S^{1} \rightarrow M_{S}$. Now assume that

$$
\alpha\left(S^{1}, \mathbf{Z}_{k}\right)^{*} \circ l_{S}^{*} \text { annihilates } \mathrm{w}_{2}\left(V_{S}-T_{S}\right) \in H_{\mathbf{Z}_{k}}^{2}\left(M ; \mathbf{Z}_{2}\right),
$$

while for $e=\frac{1}{2} p_{1}\left(V_{S}-T_{S}\right)$,

$$
\alpha\left(S^{1} ; \mathbf{Z}_{k}\right)^{*} \circ l_{S}^{*} e=\pi^{*} \circ \alpha\left(S^{1} ; 1\right)^{*} \circ l_{S}^{*} e .
$$

Then $\tau_{q}\left(M ; E_{q}(V / T)\right)$ is rigid.

Note that the relations (11.10) and (11.11) clearly follow from (11.5) by pulling back and forgetting, so that they are weaker.

Proposition 11.1 is again proved via the fixed point formula, and again the proof is most transparent where the fixed points are isolated. For simplicity we will treat that case only.

The fixed point formula asserts that

$$
\tau_{q}(M ; E)=\sum_{p} \mu_{p} \cdot f_{p}
$$

where $\mu_{p}=\prod_{i=1}^{d} \psi_{m_{i}} \varphi$ is our old friend, and where $f_{p}(q, \lambda)$ is a formal power series in $q$ with coefficients that are finite Laurent series in $\lambda$. To write down $f_{p}$, we will need the infinite product

$$
\theta(\lambda)=(1+\lambda) \cdot \prod_{n=1}^{\infty}\left(1+q^{n} \lambda\right)\left(1+q^{n} \lambda^{-1}\right),
$$

which, when expanded, defines a convergent power series in $q$ with finite Laurent series in $\lambda$ as coefficients and the domain of convergence for $\theta$ is $0<|q|<1$ and $0<|\lambda|$.

Note that

$$
\theta(q \lambda)=\lambda^{-1} \theta(\lambda)
$$

and that $\theta$ is related to our $\varphi$ by

$$
\varphi(\lambda)=\theta(\lambda) / \theta(-\lambda) .
$$


Thus $\theta$ is precisely one of the classical "theta functions" written out in our $\lambda$-parameter.

To construct $f_{p}$ from $\theta$ we need to use the exponents of the $S^{1}$ action on $T$ and $V$ at the fixed point $p$. Now the action on the tangent bundle at a fixed point $p$ is described by the integers $\left\{m_{1}, \ldots, m_{d}\right\}$, while the $S^{1}$ action on $\left.V\right|_{p}$ is described by nonzero exponents $\left\{\nu_{1}, \cdots, \nu_{\ell}\right\}$ and a subspace, $\left.V^{\prime}\right|_{p}$, on which the $S^{1}$ action is trivial.

With all of this understood, the multiplicity $f_{p}$ is now seen to be

$$
f_{p}=\lambda\left(\sum_{i} m_{i}-\sum_{i} \nu_{i}\right) / 2 \cdot \prod_{i=1}^{\ell} \psi_{\nu_{i}} \theta /\left(\prod_{i=1}^{d} \psi_{m_{i}} \theta\right) .
$$

Remark. The exponent of $\lambda$ in (11.15) is an integer by assumption (11.10). Let us spell this out a little. First note that $B \mathbf{Z}_{2}=\mathbf{R} P_{\infty}$ and that $H^{*}\left(R P_{\infty} ; \mathbf{Z}_{2}\right) \simeq$ $\mathbf{Z}_{2}[x]$ where $x$ has dimension 1 . Hence if $L=\mathbf{R}$ is the nontrivial $\mathbf{Z}_{2}$ module, of dimension 1 , on which the generator acts by -1 , then the total StiefelWhitney class $\mathrm{w}(L) \in H_{\mathbf{Z}_{2}}^{*}\left(p t ; \mathbf{Z}_{2}\right)$ is given by $1+x$.

Hence the decomposition

$$
T_{p}=\bigoplus_{i} E_{i}^{\#}
$$

implies that

$$
\mathrm{w}_{2}\left(T_{p}\right)=\prod\left(1+x m_{i}\right)^{2}=\prod\left(1+x^{2} m_{i}^{2}\right)
$$

as an element in $H_{\mathbf{Z}_{2}}^{*}\left(p ; \mathbf{Z}_{2}\right)$.

On the other hand, the map

$$
\alpha\left(S^{1}, \mathbf{Z}_{2}\right): H_{S}^{*}\left(p ; \mathbf{Z}_{2}\right) \rightarrow H_{\mathbf{Z}_{2}}^{*}\left(p ; \mathbf{Z}_{2}\right)
$$

sends $u$ to $x^{2}$. It follows that

$$
l_{S}^{*} \circ \mathrm{w}_{2}\left(T_{S}\right)=\left(\sum m_{i}^{2}\right) u \equiv\left(\sum m_{i}\right) \cdot u(\bmod 2)
$$

in $H^{2}\left(\{p\} \times B S^{1} ; \mathbf{Z}_{2}\right)$. Similarly

$$
{ }^{*} \mathrm{w}_{2}\left(T_{S}-V_{S}\right)=\sum\left(m_{i}-\nu_{i}\right) \cdot u(\bmod 2)
$$

whence (11.10) implies that $\sum\left(m_{i}-\nu_{i}\right) \equiv 0(2)$. QED

Our strategy now is to show first that the right side of (11.12) defines a meromorphic function on $T_{q^{2}}$. Then we generalize the transfer formula of $\S 7$ to argue that this meromorphic function is constant.

The multiplicity $f_{p}$ descends to $T_{q^{2}}$ if and only if $f_{p}(\lambda q)= \pm f_{p}(\lambda)$, and since (11.13) obeys $\theta(\lambda q)=\lambda^{-1} \cdot \theta(\lambda)$ we first need to understand how $\psi_{m} \theta$ transforms. Thus,

$$
\psi_{m} \theta(\lambda q)=\theta\left(\lambda^{m} q^{m}\right)=\theta\left(\lambda^{m} q^{m-1} \cdot q\right)=\lambda^{-m} q^{1-m} \cdot \theta\left(\lambda^{m} q^{m-1}\right)
$$


so that repeated iteration yields

$$
\left(\psi_{m} \theta\right)(\lambda q)=\lambda^{-m^{2}} \cdot q^{-(m)(m-1) / 2} \psi_{m} \theta .
$$

Substituted into (11.15) we therefore obtain the transformation law

$$
f_{p}(\lambda q)=\left(\lambda^{2} q\right)^{\left(\sum\left(m_{i}^{2}-\nu_{i}^{2}\right)\right) / 2} f_{p}(\lambda) \text {. }
$$

Hence $f_{p}$ descends to a meromorphic function on $T_{q^{2}}$ if and only if the exponents of $T$ and $V$ are related by

$$
\frac{1}{2} \sum\left(m_{i}^{2}-\nu_{i}^{2}\right)=0
$$

But the relation $c\left(E_{i}\right)=1+m_{i} u$ in $H_{S}^{*}(p t)$ for the total Chern class of the $E_{i}$ in (11.16) shows that

$$
p_{1}\left(E_{i}\right)=1-m_{i}^{2} u^{2}
$$

so that $\imath_{S}^{*} p_{1}\left(T_{S}\right)=\prod\left(1-m_{i}^{2} u^{2}\right)$. Hence under our assumption $f_{p}$ does descend to $T_{q^{2}}$ and our old arguments easily generalize to

Lemma 11.2. The formal power series in (11.2) defines a meromorphic function on $T_{q^{2}}$ without poles on the circle $|\lambda|=1$.

As before, the constancy of the resulting meromorphic function on $T_{q^{2}}$ is proved by checking that it has no poles on the circles $|\lambda|=|a|$ where $a \in T_{q^{2}}$ is of the form $\alpha^{s}$ for $\alpha$ some $k$ th root of $q, \alpha^{k}=q$. As before, a "transfer formula" will identify $t_{a} \tau_{q}(M ; E)$ as the character of the index of an elliptic operator on $M_{k} \subset M$; as such, it is a priori regular on the circle $|\lambda|=a$.

So, Proposition 11.1 requires us to find a transfer formula for $\tau_{q}(M ; E)$, and with this our motivation we extend $\theta$ to an operation $\theta_{a}(F)$ on complex vector bundles $F$ by the formal power series

$$
\theta_{a}(F) \equiv \Lambda_{a} F \bigotimes_{n=1}^{\infty} \Lambda_{q^{n} a} F \bigotimes_{m=1}^{\infty} \Lambda_{q^{m} a^{-1}} F^{*}, \quad|q|<a<1
$$

If $a=q^{1 / 2}$ and if $F \rightarrow X$ is a real vector bundle, introduce

$$
\theta_{q^{1 / 2}}(F)=\bigotimes_{n=1}^{\infty} \Lambda_{q^{n-1 / 2}} F
$$

Both (11.24) and (11.25) define formal power series in $q$ with coefficients in the complex or real vector bundles over $X$. Thus in $K$-theory, $\theta_{a}(F) / \theta_{-a}(F)$ agrees with $\varphi_{a}(F)$ of $\S 8$.

We will apply this construction to those vector bundles over the fixed point set $M_{k}$, which occur in the $\mathrm{Z}_{k}$-decompositions (10.1) and (10.2) of $\left.T\right|_{m_{k}}$ and $\left.V\right|_{m_{k}}$.

Our transfer formula for the cases $k$ odd and $k$ even differ and for this reason we separate them. 
Case 1. $k$ is odd. We need a word about $\operatorname{Spin}_{\mathrm{C}}$-structures, so let $X$ be a space and $E \rightarrow X$ a real, oriented vector bundle and $L \rightarrow X$ a complex line bundle. If $w_{2}(E)$ is the mod 2 reduction of $c_{1}(L)$, then $E$ can be given a Spin $_{\mathrm{C}}$-structure which is defined by the line bundle $L$ and we use $\Delta(E ; L) \rightarrow X$ to denote the corresponding $\operatorname{Spin}_{\mathrm{C}}$-bundle. That is, if we let $L^{\#}$ denote the underlying real, oriented $S O(2)$ bundle of $L$, then $E \oplus L^{\#}$ is spin, and there is a bundle of spinors, $\Delta\left(E \oplus L^{\#}\right) \rightarrow X$, which is related in $K$-theory to $\Delta(E ; L)$ by $\Delta\left(E \oplus L^{\#}\right)=\Delta(E ; L) \cdot(1+L)^{-1}$. If $E$ is already spin, then $c_{1}(L) \equiv 0(\bmod$ 2 ) and $L$ has a square root, in which case one can choose a spin structure on $E$ and a square root $L^{1 / 2}$ of $L$ such that $\Delta(E ; L)=\Delta(E) \otimes L^{-1 / 2}$ where $\Delta(E)$ is the bundle of spinors for the spin structure on $E$.

Our present situation when $k$ is odd provides examples. Since $M$ is spin,

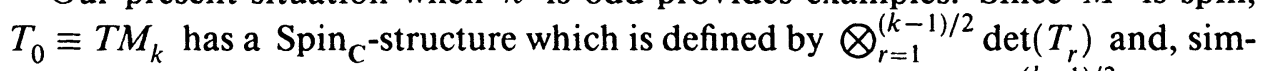

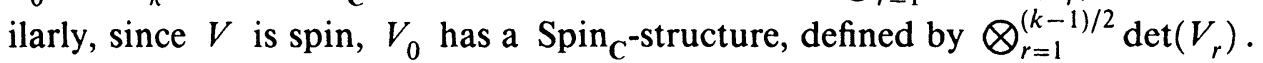

With this understood, we need one last fact before we can write down the $k$ odd transfer formula. Let then $s$ be relatively prime to $k$ and introduce representatives $\omega(r)$ in $\{1, \cdots,(k-1) / 2\}$ for the $\bmod k$ reductions of $s \cdot r$. Then we have

Lemma 11.3. If $k$ is odd and the assumptions of Proposition 11.1 hold, then the complex line bundle

$$
\nu_{s}=\bigotimes_{r=1}^{(n-1) / 2} \operatorname{det}\left(V_{r}\right)^{\omega(r)} \cdot \operatorname{det}\left(T_{r}\right)^{-\omega(r)}
$$

admits a kth root $\nu_{s}^{1 / k}$ over $M_{k}$.

We defer our proof of this fact temporarily so that we can write down the transfer formula

$$
\begin{aligned}
& t_{\alpha^{s}} \tau_{q}(M ; E)=\tau_{q}\left(M_{k}^{\prime} ; \alpha^{\sigma} \cdot \nu_{s}^{1 / k} \otimes\left[\frac{\Delta\left(V_{0} ; \bigotimes_{r=1}^{(k-1) / 2} \operatorname{det}\left(V_{r}\right)\right)}{\Delta\left(T_{0} ; \bigotimes_{r=1}^{(k-1) / 2} \operatorname{det}\left(T_{r}\right)\right)} \otimes E_{0}\right]\right. \\
& \left.\bigotimes_{r=1}^{(k-1) / 2} \theta_{\alpha^{\omega(r)}}\left(V_{r}\right) \bigotimes_{r=1}^{(k-1) / 2} \theta_{\alpha^{\omega(r)}}\left(T_{r}\right)^{-1}\right)
\end{aligned}
$$

where $E_{0}=\left(\bigotimes_{n=1}^{\infty} \Lambda_{q^{n}} V_{0}\right) \otimes\left(\bigotimes_{n=1}^{\infty} \Lambda_{q^{n}} T_{0}\right)^{-1}$. Here,

$$
\sigma=\frac{1}{2} \sum_{r=1}^{(k-1) / 2}\left(\frac{\omega(r)^{2}}{k}-\omega(r)\right)\left(\operatorname{dim}_{\mathbf{C}} V_{r}-\operatorname{dim}_{\mathbf{C}} T_{r}\right)
$$

is constant on a connected component of $M_{k}$, and as before $M_{k}^{\prime}$ denotes $M_{k}$ in the orientation given by (7.5). 
Let us remark that $\sigma$ is a whole number under Proposition 11.1's assumptions. Indeed, as $k$ is odd, one need only verify that

$$
b \equiv \sum_{r=1}^{(k-1) / 2}\left(\operatorname{dim}_{\mathbf{C}} V_{r}-\operatorname{dim}_{\mathbf{C}} T_{r}\right) \omega(r)^{2}
$$

is divisible by $k$. Then the integer $2 \sigma$ is of the form $\frac{1}{k} \times$ even and hence even. To verify $b / k$ 's integrality, we fix $p \in M_{k}$ and introduce the section $q_{p}: B \mathbf{Z}_{k} \rightarrow\{p\} \times B \mathbf{Z}_{k} \hookrightarrow M_{\mathbf{Z}_{k}}$ and calculate

$$
q_{p}^{*} \alpha\left(S^{1} ; \mathbf{Z}_{k}\right)^{*} \imath_{S}^{*} p_{1}\left(V_{S}-T_{S}\right)=-b \cdot u_{k}
$$

where $u_{k} \in H^{2}\left(B \mathbf{Z}_{k} ; \mathbf{Z}\right) \simeq \mathbf{Z}_{k}$ is the generator of $H^{*}\left(B \mathbf{Z}_{k} ; \mathbf{Z}\right) \simeq \mathbf{Z}\left[u_{k}\right] /\left(k \cdot u_{k}\right)$. Since (11.27) must vanish, by (11.11), we have $b \equiv 0(\bmod k)$ as claimed.

To verify (11.26), we first examine the effect of translation by $\alpha^{s}$ on the left side of (11.26) by using the fixed point formula on $M$ to equate $\tau_{q}(M ; E)$ with the right side of (11.12). Our computation of the $\alpha^{s}$ translation of the right side of (11.12) is facilitated by more than one use of the identity $\theta(\lambda q)=\lambda^{-1} \theta(\lambda)$; in particular, use of this identity gives

$$
t_{\alpha^{s}} \psi_{m} \theta=\left(\lambda^{m} \alpha^{\omega}\right)^{-\ell} q^{-1 / 2\left(\ell^{2}-\ell\right)} \psi_{m} t_{\alpha^{(v}} \theta
$$

where $\omega \in\{1, \ldots, k-1\}$ and $\ell \in \mathbf{Z}$ are determined by the formula $s \cdot m=$ $\ell \cdot k+\omega$.

We will use (11.28) after introducing $\left(\ell_{i}, \omega_{i}\right)$ and $\left(\ell_{i}^{\prime}, \omega_{i}^{\prime}\right)$ in $\mathbf{Z} \times$ $\{1, \cdots, k-1\}$ by the formulae $s \cdot m_{i}=\ell_{i} \cdot k_{i}+\omega_{i}$ and $s \cdot \nu_{i}=\ell_{i}^{\prime} \cdot k_{i}+\omega_{i}^{\prime}$. Then we find that

$$
t_{\alpha^{s}} f_{p}=\lambda^{a} \alpha^{b} \lambda^{\left(\sum m_{i}-\sum \nu_{i}\right) / 2} \cdot\left(\prod_{i=1} \psi_{\nu_{i}} t_{\alpha^{\omega_{i}^{\prime}}} \theta\right) \cdot\left(\prod_{i=1} \psi_{m_{i}} t_{\alpha^{\omega_{i}}} \theta\right)^{-1},
$$

where $a=\sum \ell_{i}^{\prime} \nu_{i}-\sum \ell_{i} m_{i}$ and

$$
b=-\sum\left(\frac{1}{2} k\left(\ell_{i}^{\prime 2}-\ell_{i}^{\prime}\right)+\omega_{i}^{\prime} \ell_{i}^{\prime}+\frac{1}{2} s \nu_{i}\right)+\frac{1}{2} \sum\left(\frac{1}{2} k\left(\ell_{j}^{2}-\ell_{j}\right)+\omega_{j} \ell_{j}+\frac{1}{2} s m_{j}\right) .
$$

If we use (11.29) and (11.30) with (7.10) (which gives us $t_{\alpha^{s}} \mu_{p}$ ), then we can sum over the fixed points to obtain a formula for the $\alpha^{s}$ translate of $\tau_{q}(M, E)$. To prove (11.26), we will identify the resulting expression with the formula for the right side of (11.26), which is given to us by the fixed point theorem applied on $M_{k}$. With the contents of $\S 7$ well digested, the task requires only the identification of (11.29) as the formula for the $S^{1}$-module structure of

$$
\begin{gathered}
\alpha^{\sigma} \cdot \nu_{s}^{1 / k} \otimes\left[\frac{\Delta\left(V_{0} ; \bigotimes_{r=1}^{(k-1) / 2} \operatorname{det}\left(V_{r}\right)\right)}{\Delta\left(T_{0} ; \bigotimes_{r=1}^{(k-1) / 2} \operatorname{det}\left(T_{r}\right)\right)} \otimes E_{0}\left(V_{0}, T_{0}\right)\right] \\
\bigotimes_{r=1}^{(k-1) / 2}\left(\theta_{\left.\alpha^{\omega(r)}\left(V_{r}\right) \otimes \theta_{\alpha^{\omega(r)}}\left(T_{r}\right)^{-1}\right)}\right.
\end{gathered}
$$

at the fixed point $p$. 
To make the requisite identification, we begin with the equality $\sum_{i} m_{i}^{2}=$ $\sum_{j} \nu_{j}^{2}$ which implies that the numbers $b$ in (11.30) and $\sigma$ in (11.31) are the same. Also, this same equality shows that $\lambda^{a}$ describes the complex line $\left.\nu_{s}^{1 / k}\right|_{p}$ as an $S^{1}$-module. Meanwhile, the $S^{1}$ module structure of

$$
\Delta\left(V_{0} ; \bigotimes_{r=1}^{(k-1) / 2} \operatorname{det}\left(V_{r}\right)\right) \otimes \Delta\left(T_{0} ; \bigotimes_{r=1}^{(k-1) / 2} \operatorname{det}\left(T_{r}\right)\right)^{-1} \otimes E_{0} l_{p}
$$

is given by

$$
\lambda^{\left(\sum m_{i}-\sum \nu_{j}\right) / 2} \cdot \prod_{\substack{i: m_{i} \\ j: \nu_{j}} 00(\bmod k)} \psi_{\nu_{i}} \theta \cdot\left(\psi_{m_{i}} \theta\right)^{-1},
$$

while that of $\left.\theta_{\left.\alpha^{\omega(r)}\right)}\left(T_{r}\right)\right|_{p}$ is given by $\prod_{l: m_{i}} \equiv r(\bmod k) \psi_{m_{i}} t_{\alpha^{\omega_{i}}} \theta$ and that of $\left.\theta_{\alpha^{\omega(r)}}\left(V_{r}\right)\right|_{P}$ is given by $\prod_{t: \nu_{i}} \equiv r(\bmod k) \psi_{\nu_{i}} t_{\alpha}^{\omega_{i}^{\prime}} \theta$. Thus, we have identified (11.29) as giving the $S^{1}$-module structure of (11.31) at $p$ and, modulo Lemma 11.3, we are finished with establishing (11.26).

Case 2. $k$ is even. When $k$ is even, (11.26) gets modified and to write down the modification we need the $k$ even analog of Lemma 11.3.

Lemma 11.4. If the assumptions of Proposition 11.1 hold and if $k$ is even and $s$ is relatively prime to $k$, then

(1) The complex line bundle

$$
\nu_{s} \equiv \bigotimes_{r=1}^{k / 2-1}\left[\left(\operatorname{det}\left(V_{r}\right)\right)^{\omega(r)} \otimes\left(\operatorname{det}\left(T_{r}\right)\right)^{-\omega(r)}\right]
$$

has a $\left(\frac{k}{2}\right)$ th root, $\nu_{s}^{2 / k} \rightarrow M_{k}$.

(2) The vector bundle $T_{0} \oplus V_{0} \rightarrow M_{k}$ has a Spin $_{\mathrm{C}}$ structure which is defined by the line bundle

$$
\omega \equiv \bigotimes_{r=1}^{k / 2-1} \operatorname{det}\left(V_{r}\right) \bigotimes_{r=1}^{k / 2-1}\left(\operatorname{det}\left(T_{r}\right)\right)^{-1} \otimes \nu_{s}^{2 / k}
$$

We will again postpone the proof of Lemma 11.4 and write down the transfer formula in the case where $k$ is even:

(11.33)

$$
\begin{aligned}
& t_{\alpha^{s}} \tau_{q}(M ; E) \\
& =\tau_{q}\left(M_{k}^{\prime}, \alpha^{\sigma} \cdot \nu_{s}^{2 / k} \otimes\left[\frac{\Delta\left(V_{0} \oplus T_{0} ; \omega\right)}{\Delta\left(T_{0} \oplus T_{0}\right)} \otimes E_{0}\right] \bigotimes_{r=1}^{k / 2} \theta_{\alpha^{\omega(r)}}\left(V_{r}\right) \bigotimes_{r=1}^{k / 2} \theta_{\alpha^{(\omega(r)}}\left(T_{r}\right)^{-1}\right),
\end{aligned}
$$

where $E_{0}$ is the same as before, but on a connected component of $M_{k}$,

$$
\sigma=\frac{1}{2} \sum_{r=1}^{k / 2-1}\left(\frac{\omega(r)^{2}}{k}-\omega(r)\right) \cdot\left(\operatorname{dim}_{\mathrm{C}} V_{r}-\operatorname{dim}_{\mathrm{C}} T_{r}\right) .
$$


Under our assumptions, $\sigma$ is again an integer.

The proof of (11.33) goes as before, so we end this section with the proofs of Lemmas 11.3 and 11.4 .

Proof of Lemma 11.3. Our result is obtained by studying the characteristic classes of $\alpha\left(S^{1} ; \mathbf{Z}_{k}\right)^{*}\left(V_{S}-T_{S}\right)$ when restricted to $M_{k} \times B Z_{k}$. These are easy to compute because

$$
\alpha\left(S^{1} ; \mathbf{Z}_{k}\right)^{*} l_{S}^{*} V_{S}=V_{0} \bigoplus_{r=1}^{(k-1) / 2}\left(V_{r} \otimes_{\mathbf{C}} L^{n}\right)
$$

where $L \rightarrow B \mathbf{Z}_{k}$ is the complex line bundle $\left(E \mathbf{Z}_{k} \times{ }_{\mathbf{Z}_{k}} \mathbf{C}\right)$ whose first Chern class is $u_{k}$, the generator of $H^{2}\left(B \mathbf{Z}_{k} ; \mathbf{Z}\right) \simeq \mathbf{Z}_{k}$. The bundle $T$ has a similar decomposition, so that the restriction to $M_{k} \times B Z_{k}$ of $\alpha\left(S^{1} ; \mathbf{Z}_{k}\right)^{*} \frac{1}{2} p_{1}\left(V_{S}-T_{S}\right)$ has a component in $H^{2}\left(M_{k}\right) \otimes H^{2}\left(B Z_{k}\right)$, given by

$$
\sum_{r=1}^{(k-1) / 2} r \cdot\left(c_{1}\left(V_{r}\right)-c_{1}\left(T_{r}\right)\right) \cdot u_{k}
$$

But the assumption (11.11) implies that this restriction, i.e., $\alpha\left(S^{1} ; \mathbf{Z}_{k}\right) \circ l_{S}^{*} e$, be in the image of $\pi^{*}$ and hence have no mixed term whatsoever. This implies that $c_{1}\left(\nu_{S}\right)=\sum_{r=1}^{(n-1) / 2} r\left(c_{1}\left(V_{r}\right)-c_{2}\left(T_{r}\right)\right)$ is divisible by $k$. QED

Proof of Lemma 11.4. When $k$ is even, the restriction to $M_{k} \times B Z_{k}$ of $\alpha\left(S^{1} ; \mathbf{Z}_{k}\right)^{*} V_{S}$ is

$$
\alpha\left(S^{1} ; \mathbf{Z}_{k}\right)^{*}{ }^{*}{ }_{S}^{*} V_{S}=V_{0} \bigoplus_{r=1}^{(k-1) / 2}\left(V_{r} \otimes_{\mathbf{C}} L^{r}\right) \oplus\left(V_{k / 2} \otimes \widehat{L}\right)
$$

where $L^{r}$ is as in (11.34), and where $\widehat{L} \rightarrow B Z_{k}$ is the real line bundle whose complexification is $L^{k / 2}$. The restriction of $\alpha\left(S^{1} ; Z_{k}\right)^{*} i_{S}^{*} \frac{1}{2} p_{1}\left(V_{S}-T_{S}\right)$ to $M_{k} \times$ $B Z_{k}$ has one component in $H^{2}\left(M_{k}\right) \otimes H^{2}\left(B Z_{k}\right)$ :

$$
\sum_{r=1}^{k / 2-1} r \cdot\left(c_{1}\left(V_{r}\right)-c_{1}\left(T_{r}\right)\right) \cdot u_{k}+\omega_{2}\left(V_{k / 2}-T_{k / 2}\right) \cdot\left(\frac{k}{2}\right) \cdot u_{k} \text {. }
$$

(The calculation is straightforward, though it uses Lemma 10.1 to insure that $V_{k / 2}$ is orientable and Lemma 9.4 to insure that it is even dimensional.)

The assumptions in Proposition 11.1 require this class to vanish, a condition that demands the divisibility of $\sum_{r=1}^{k / 2-1} r \cdot\left(c_{1}\left(V_{r}\right)-c_{1}\left(T_{r}\right)\right)$ by $\frac{k}{2}$ in $H^{2}\left(M_{k} ; \mathbf{Z}\right)$. Now we have proved our first assertion because $c_{1}\left(\nu_{s}\right)$ and $\sum_{r=1}^{k / 2-1} r \cdot\left(c_{1}\left(V_{r}\right)-\right.$ $\left.c_{1}\left(T_{r}\right)\right)$ are equal mod $k$.

The second assertion of Lemma 11.4 also follows from the vanishing of (11.37) since (11.37) can vanish only if

$$
\left(\sum_{r=1}^{h / 2-1} r \cdot\left(c_{1}\left(V_{r}\right)-c_{1}\left(T_{r}\right)\right)\right)_{\bmod 2}=\mathrm{w}_{2}\left(V_{k / 2}-T_{k / 2}\right),
$$


and when (11.38) holds, the line bundle $\omega$ in (11.32) has second StiefelWhitney class

$$
c_{2}(\omega)_{\bmod 2}=\sum_{r=1}^{k / 2-1}\left(c_{2}\left(V_{r}\right)-c_{2}\left(T_{r}\right)\right)_{\bmod 2}=\mathrm{w}_{2}\left(V_{k / 2}-T_{k / 2}\right) .
$$

Now $V$ and $T$ are separately spin, and $\left\{V_{i}, T_{i}\right\}_{i=0}^{k / 2}$ are all orientable, so the right side of (11.39) equals $\mathrm{w}_{2}\left(V_{0}-T_{0}\right)=\mathrm{w}_{2}\left(V_{0}+T_{0}\right)$ and thus the second assertion is proved.

\section{The Dirac operator}

Our presentation so far has concerned only the signature operator, but Witten has also conjectured various rigidity theorems for twists of the Dirac operator. We will show here how Witten's other conjectures follow. In [W], Witten has twisted the Dirac operator with representations $\left\{R_{n}^{\prime}\right\}_{n=0}^{\infty}$ which are defined by the formal power series in the parameter $q^{1 / 2}$ :

$$
R_{q}^{\prime}(T) \equiv \sum_{n=1}^{\infty} R_{n}^{\prime} q^{n / 2}=\bigotimes_{n=0}^{\infty} \Lambda_{q^{n+1 / 2}} T \bigotimes_{m=1}^{\infty} S_{q^{m}} T \text {, }
$$

and he conjectured that when $S^{1}$ acts by isometries on a spin manifold $M$,

$$
\tau_{q}^{\prime}(M) \equiv \mathrm{ch} \cdot \operatorname{ind}\left(\not \partial \otimes R_{q}^{\prime}(T)\right)
$$

is rigid.

Witten also considered generalizing $(12.1)$ by fixing a real, oriented vector bundle $V \rightarrow M$ and defining

$$
E_{q}^{\prime}(V / T) \equiv\left(\bigotimes_{n=1}^{\infty} \Lambda_{q^{n-1 / 2}} V\right)\left(\bigotimes_{n=1}^{\infty} \Lambda_{q^{n-1 / 2}} T\right)^{-1}
$$

Then if $S^{1}$ acts compatibly on $V$ and if the equivariant characteristic classes of $V$ are suitably restricted, Witten conjectures that

$$
\tau_{q}^{\prime}\left(M ; E^{\prime}(V / T)\right) \equiv \mathrm{ch} \cdot \operatorname{ind}\left(\not \partial \otimes R_{q}^{\prime}(T) \otimes E_{q}^{\prime}(V / T)\right)
$$

is also rigid. The precise assertion is

Proposition 12.1. Let $M$ be a smooth, oriented, compact spin manifold on which $S^{1}$ acts isometrically. Then $\tau_{q}^{\prime}(M)$ is rigid. Also, let $V \rightarrow M$ be a real, oriented vector bundle with $\mathrm{w}_{2}(V)=0$ on which $S^{1}$ acts compatibly, and assume Proposition 11.1 's restrictions on the equivariant characteristic classes of $T-V$. Then $\tau_{q}^{\prime}\left(M ; E^{\prime}(V / T)\right)$ is rigid.

Proposition 12.1 will be proved in the manner of our analysis for $\tau_{q}(M)$ (and $\left.\tau_{q}(M ; E(V / T))\right)$, though for brevity's sake we will restrict our attention here to $\tau_{q}^{\prime}(M)$ and leave the generalization to $\tau_{q}^{\prime}\left(M, E^{\prime}(V / T)\right)$ for our capable readers. 
The introduction of a covering space for our $q$-space, the punctured, open unit disc $D^{0} \backslash\{0\}$, is required in order for us to interpret the half integral powers of $q$ in (12.1). A double cover would suffice, but it is convenient to consider the universal covering space of $D^{0} \backslash\{0\}$ which is the open upper half plane $H$. So allow us now to digress to consider complex tori parametrized by points in $H$.

The upper half plane parametrizes complex tori in the following way. We pick a standard torus $\mathbf{R}^{2} / \mathbf{Z} \times \mathbf{Z}$ with basis Euclidean coordinates $(x, y)$ and then use $\tau \in H$ to define the complex parameter $z_{\tau} \equiv x+y \cdot \tau$; the resulting complex manifold we call $T_{\tau}$. The projection from $H$ to $D^{0} \backslash\{0\}$ sends $\tau$ to $q=e^{2 \pi i \tau}$ and identifies $T_{\tau}$ with $T_{q}=\mathrm{C}^{*} / q$ via the exponential map which sends $z_{\tau} \in \mathbf{C}$ to $\lambda=e^{2 \pi i z_{\tau}} \in \mathbf{C}^{*}$. For those who are more comfortable with complex tori that are defined as $\mathbf{C} /$ lattice, use the linear transformation

$$
\left(\begin{array}{ll}
1 & \operatorname{Re} \tau \\
0 & \operatorname{Im} \tau
\end{array}\right): \mathbf{R}^{2} \rightarrow \mathbf{R}^{2}
$$

to define a complex analytic diffeomorphism from $T_{\tau}$ to $\mathbf{C} / \mathbf{Z} \times \tau \cdot \mathbf{Z}$.

It is a well-known fact that $S L(2, \mathbf{Z})$ acts on $H$ and so on our space of complex tori, $\left\{T_{\tau}\right\}_{\tau \in H}$. This action can be made explicit by introducing the linear isomorphisms

$$
e_{1} \equiv\left(\begin{array}{ll}
1 & 1 \\
0 & 1
\end{array}\right) \text { and } e_{2}=\left(\begin{array}{cc}
0 & 1 \\
-1 & 0
\end{array}\right)
$$

of $\mathbf{R}^{2}$. Then $\left\{e_{1}, e_{2}\right\}$ preserve $\mathbf{R}^{2} / \mathbf{Z} \times \mathbf{Z}$, they generate $S L(2, \mathbf{Z})$, and

$$
e_{1}^{*} z_{\tau}=z_{\tau+1}, \quad e_{2}^{*} z_{\tau}=-\tau \cdot z_{-1 / \tau}
$$

so $e_{1}$ and $e_{2}$ define complex analytic isomorphisms

$$
e_{1}: T_{\tau} \stackrel{\sim}{\rightarrow} T_{\tau+1}, \quad e_{2}: T_{\tau} \sim \underset{\rightarrow}{\sim} T_{-1 / \tau} .
$$

Thus, our original parameter space, $D^{0} \backslash\{0\}$, is given as $H / \mathbf{Z} \cdot e_{1}$.

Now recall that our basic object $\varphi_{q}$ of (5.3) defines, by virtue of (5.4), a meromorphic function on $\mathbf{C}^{*} / q^{2}$. We went to the $q^{2}$-torus to avoid facing the minus sign in (5.4). To interpret $\varphi_{q}$ on the torus $\mathbf{C}^{*} / q$ and by pullback on $T_{\tau}$, we must come to grips with that minus sign. We do so by introducing the flat line bundle

$$
L_{\tau} \equiv \mathbf{R}^{2} \times \mathbf{C} / \mathbf{Z} \times \mathbf{Z} \rightarrow T_{\tau}
$$

where $\mathbf{Z} \times \mathbf{Z}$ acts on $\mathbf{R}^{2}$ above by translation and on $\mathbf{C}$ by sending $\{k, \ell\} \in$ $\mathbf{Z} \times \mathbf{Z}$ and $v \in \mathbf{C}$ to $(-1)^{\ell} \cdot v$. Clearly, $e_{1}^{*} L_{\tau+1}=L_{\tau}$ so $L_{\tau}$ is the pullback from $\mathbf{C}^{*} / q$ of a flat line bundle $L_{q}$ of which $\varphi$ is a meromorphic section. Thus, $\varphi$ pulls back to $T_{\tau}$ as a meromorphic section, $\varphi_{\tau}$, of $L_{\tau}$ which obeys $e_{1}^{*} \varphi_{\tau+1}=\varphi_{\tau}$. 
Up to isomorphism, there are four flat line bundles over $T_{\tau}$ that have trivial square. This is because $H^{1}\left(T_{\tau} ; \mathbf{Z}_{2}\right) \simeq \mathbf{Z}_{2} \times \mathbf{Z}_{2}$. The trivial bundle is one, $L_{\tau}$ is another, and a third, which we will call $L_{\tau}^{\prime \prime}$, is defined as in (12.7) but with the action of $\mathbf{Z} \times \mathbf{Z}$ on $\mathbf{C}$ sending $\{k, \ell\}$ and $v \in \mathbf{C}$ to $(-1)^{k} \cdot v$. The fourth is $L_{\tau}^{\prime} \simeq L_{\tau} \otimes L_{\tau}^{\prime \prime}$.

The group $S L(2, \mathrm{Z})$ permutes these line bundles since

$$
e_{2}^{*} L_{-1 / \tau}=L_{\tau}^{\prime \prime}, \quad e_{1}^{*} L_{\tau+1}^{\prime \prime}=L_{\tau}^{\prime} .
$$

With all of this understood, we return to our spin manifold $M$ with $S^{1}$ action and observe that the transfer formulae (7.5) and (7.12) imply that $\tau_{q}(M)$ pulls back to $T_{\tau}$ as a meromorphic section, $\mathscr{S}_{\tau}$, of $L_{\tau}^{\varepsilon}$, with $\varepsilon=0$ if the $S^{1}$ action lifts to the spin bundle and with $\varepsilon=+1$ if it does not. There is a similar interpretation for $\tau_{q}^{\prime}(M)$.

Lemma 12.2. Let $M$ be a spin manifold on which $S^{1}$ acts, write $q=e^{2 \pi i \tau}$ for $\tau \in H$, and write $\lambda=e^{2 \pi i z_{\tau}}$ for $z_{\tau}=x+\tau y \in \mathbf{C}$. Then $\tau_{q}^{\prime}(M)$ of $(12.1)$ is naturally defined over $H$ as a family of meromorphic sections

$$
\left\{\mathscr{S}_{\tau}^{\prime}: T_{\tau} \rightarrow\left(L_{\tau}^{\prime}\right)^{\varepsilon}\right\}_{\tau \in H}
$$

Our proof of Proposition 12.1 will show more or less directly that $\mathscr{S}_{\tau}^{\prime}$ has no poles. (There is another proof of Proposition 12.1 which establishes that $\mathscr{S}_{\tau}^{\prime}$ and $e_{1}^{*} e_{2}^{*}\left(\mathscr{S}_{-1 /(\tau+1)}\right)$ are a priori proportional, and since $\mathscr{S}_{-1 /(\tau+1)}$ is now known to be rigid, the rigidity of $\mathscr{S}_{\tau}^{\prime}$ is finally deduced. This argument, in the isolated fixed point case only, is given in the next section.)

We will prove Lemma 12.2 with the help of the fixed point formula for the Dirac operator, but to properly interpret this formula, we must digress to consider some special sections of $L_{\tau}^{\prime}$. For this purpose, fix $\tau \in H$ and $q=e^{2 \pi i \tau}$ and set $q^{1 / 2}=e^{\pi i \tau}$. For $\xi \in \mathbf{C}$, introduce $\lambda=e^{2 \pi i \xi}$ and $\lambda^{1 / 2}=e^{\pi i \xi}$, and then define

$$
\chi_{\tau}(\xi)=\frac{\lambda^{1 / 2}}{1-\lambda} \cdot \prod_{n=1}^{\infty} \frac{\left(1+q^{n} q^{-1 / 2} \lambda\right)\left(1+q^{n} q^{-1 / 2} \lambda^{-1}\right)}{\left(1-q^{n} \lambda\right)\left(1-q^{n} \lambda^{-1}\right)} .
$$

As a function on $\mathbf{C}, \chi_{\tau}$ is observedly meromorphic, and it obeys

$$
\chi_{\tau}(\xi+1)=-\chi_{\tau}(\xi) \text { and } \chi_{\tau}(\xi+\tau)=-\chi_{\tau}(\xi) \text {. }
$$

Thus, as a function of the complex coordinate $\xi \equiv z_{\tau}$, we see $\chi_{\tau}$ descending to the $\tau$-torus $T_{\tau}$ as a meromorphic section of $L_{\tau}^{\prime}$. In a sense, it is our basic section in that it has the minimal number of poles and zeros on $T_{\tau}$-one pole at $z_{\tau}=0$ and one zero at $z_{\tau}=\frac{1}{2}(1+\tau)$.

Now by iterating $(12.10)$, we see that $\left(\psi_{m} \chi_{\tau}\right)\left(z_{\tau}\right) \equiv \chi_{\tau}\left(m \cdot z_{\tau}\right)$ defines, for integer $m$, either a meromorphic section of $L_{\tau}^{\prime}$ (if $m$ is odd) or a meromorphic function on $T_{\tau}$ (if $m$ is even). Of course $\psi_{m} \chi_{\tau}$ has $|m|$ poles and $|m|$ zeros. 
Additional meromorphic sections of $L_{\tau}^{\prime}$ can be constructed if we remember that the meromorphic sections of $L_{\tau}^{\prime}$ form a module for the ring of meromorphic functions on $T_{\tau}$. Of course, the product of two meromorphic sections is a meromorphic function.

At this point, we have enough meromorphic sections of $L_{\tau}^{\prime}$ to identify $\tau_{q}^{\prime}(M)$, at least when $S^{1}$ acts with isolated fixed points, because the fixed point formula for $\not \partial \otimes R_{q}^{\prime}$ is summarized by

Lemma 12.3. Let $S^{1}$ act on $M$ by isometry with isolated fixed points. Write $q=e^{2 \pi i \tau}$ for $\tau \in H$. Then the fixed point formula applied to $\not \partial \otimes R_{q}^{\prime}$ yields

$$
\operatorname{ch} \cdot \operatorname{ind}\left(\not \partial \otimes R_{q}^{\prime}\right)\left(e^{i \theta}\right)=\sum_{\{p\}} \mu_{p}^{\prime}\left(e^{i \theta}\right)
$$

where

$$
\mu_{p}^{\prime}\left(e^{i \theta}\right)=\left.\prod_{i=1}^{d}\left(\psi_{m_{i}} \chi_{\tau}\right)\right|_{z_{\tau}=\theta} .
$$

To prove the lemma, we return to (3.7) to get the multiplicity for $\not \partial$ and then use (4.1) to generalize to $\not \partial \otimes R_{q}^{\prime}$.

Note that the lemma exhibits the multiplicity at an isolated fixed point $p$ as a meromorphic section of $\left(L_{\tau}^{\prime}\right)^{\sum_{i} m_{i}}$. Also, Lemma 9.3 insures that $\left(\sum_{i} m_{i}\right)_{\bmod 2}$ is the same at each fixed point, so when $S^{1}$ acts with only isolated fixed points, Lemma 12.3 identifies $\tau_{q}^{\prime}(M)$ as a meromorphic section of $\left(L_{\tau}^{\prime}\right)^{\varepsilon}$, as claimed in Lemma 12.2.

Now suppose that $S^{1}$ acts with fixed point sets $\{P\}$, not necessarily points. To understand how to generalize Lemma 12.3, consider first just the fixed point formula for the Dirac operator-no twists. For this, remember that the normal bundle to $P$ decomposes as

$$
N_{P}=\bigoplus_{i} E_{i}^{\#}
$$

with $E_{i}^{\#} \rightarrow P$ being the realization of a complex vector bundle on which $S^{1}$ acts by $\lambda \rightarrow \lambda^{m_{i}}$. We will use (12.11) to define the orientation on $N_{P}$ and hence $T_{P}$ when $\operatorname{dim} P>0$. Also, since $M$ is spin, the complex line bundle

$$
\operatorname{det} N_{P}=\bigotimes_{i} \operatorname{det} E_{i}
$$

makes $T P$ a $\operatorname{Spin}_{\mathrm{C}}$-bundle so we can introduce the bundle of spinors $\Delta\left(T P ; \operatorname{det} N_{P}\right) \rightarrow P$.

With this understood, the fixed point formula for the Dirac operator reads

$$
\mathrm{ch} \cdot \text { ind } \not \partial=\sum_{\{P\}} \mu_{P, \not}
$$


with

$$
\mu_{P, \varnothing}=\mathrm{ch} \cdot \operatorname{ind}\left(d_{S}^{P} \otimes \Delta\left(T P ; \operatorname{det} N_{P}\right)^{-1} \bigotimes_{i} \lambda^{m_{i} / 2}\left(\Lambda_{-\lambda^{m_{i}}} E_{i}\right)^{-1}\right),
$$

where $d_{S}^{P}$ is the signature operator on $P$.

Let us remark here that $d_{S}^{P} \otimes \Delta\left(T P ; \operatorname{det} N_{P}\right)^{-1}$ plays the role of the Dirac operator on $P$, for if $P$ should be a bona fide spin manifold, we can use (2.11) to write

$$
d_{S}^{P} \otimes \Delta\left(T P ; \operatorname{det} N_{P}\right)^{-1}=\not \partial^{P} \otimes \operatorname{det} N_{P}^{1 / 2} .
$$

To step from (12.14) to the fixed point formula for $\not \partial \otimes R_{q}^{\prime}$, let us introduce a generalization of $\not \partial \otimes R_{q}^{\prime}$ to an oriented manifold $X$ whose tangent bundle has a Spin $_{\mathrm{C}}$-structure from a complex line bundle, $L \rightarrow X$. Thus we set

$$
\tau_{q}^{\prime}\left(X_{L}\right)=\mathrm{ch} \cdot \operatorname{ind}\left(d_{S} \otimes \Delta(T X ; L)^{-1} \otimes R_{q}^{\prime}(T X)\right)
$$

Further generalize (12.16) so that when $E_{q}$ is a formal power seres in $q$ with coefficients in $K(X)$, we have

$$
\tau_{q}^{\prime}\left(X_{L} ; E\right)=\mathrm{ch} \cdot \operatorname{ind}\left(d_{S} \otimes \Delta(T X ; L)^{-1} \otimes R_{q}^{\prime}(T X) \otimes E_{q}\right) .
$$

Remark now that when $E_{q}$ is a finite sum of convergent power series in $q$ on $0 \leq|q|<1$ with coefficients in $K(X)$, then so is $R_{q}^{\prime}(T X) \otimes E_{q}$. This can be seen by writing $T X$ as constant + nilpotent in $K(X)$.

Before returning to $\not \partial \otimes R_{q}^{\prime}$, let us introduce one extra bit of notation. Let $E$ be a complex vector bundle over a space $X$. Then write $\lambda^{1 / 2}=e^{\pi i z}$ for $z \in \mathbf{C}$, and define

$$
\widehat{\chi}_{\tau}(E)(z)=\lambda^{1 / 2}\left(\Lambda_{-\lambda} E\right)^{-1} \bigotimes_{m=1}^{\infty}\left(\Lambda_{\lambda q^{m-1 / 2}} E \otimes \Lambda_{\lambda-1 q^{m-1 / 2}} E^{*}\right) \bigotimes_{m=1}^{\infty}\left(S_{\lambda q^{m}} E \otimes S_{\lambda-1} q^{m} E^{*}\right)
$$

which is a formal power series in $q^{1 / 2}$ whose coefficients are vector bundles $\otimes$ finite Laurent series in $\lambda^{1 / 2}=e^{i \pi z}$. However, when $E$ is a line bundle, we can introduce as in $\S 8$ the nilpotent $\log E \in K(X)$ and then we have in $K(X)$ the identity

$$
\widehat{\chi}_{\tau}(E)(z)=\sum_{k \geq 0} \widehat{\chi}_{\tau}^{(k)}(z) \cdot \frac{1}{k !}(\log E)^{k}
$$

where

$$
\hat{\chi}_{\tau}^{(k)}=e^{i \pi z}\left(\frac{d}{d z}\right)^{k}\left(e^{-i \pi z} \chi_{\tau}\right)
$$

with $\chi_{\tau}$ as defined in (12.9).

Therefore, when $E$ is a line bundle, we can view $\hat{\chi}_{\tau}(E)$ as an element of the tensor product of $K(X)$ with the meromorphic sections over $T_{\tau}$ of $L_{\tau}^{\prime}$. If 
$E$ is a sum of line bundles $L_{1} \oplus \cdots \oplus L_{k}$, then we have $\widehat{\chi}_{\tau}(E)=\otimes \widehat{\chi}_{\tau}\left(L_{k}\right)$ and so, by the splitting principle, we can, for general $E$, view $\hat{\chi}_{\tau}(E)$ as an element of $K(X) \otimes_{\mathrm{Z}}$ \{meromorphic sections over $T_{\tau}$ of $\left(L_{\tau}^{\prime}\right)^{\operatorname{dim}_{\mathrm{c}}{ }^{2}}$ \}.

With all of this understood, the fixed point formula for $\tau_{q}^{\prime}(M)$ reads

$$
\mathrm{ch} \cdot \operatorname{ind}\left(\not \partial \otimes R_{q}^{\prime}\right)=\sum_{\{P\}} \tau_{q}^{\prime}\left(P_{\operatorname{det} N_{P}} ; \bigotimes_{i} \psi_{m_{i}} \widehat{\chi}_{\tau}\left(E_{i}\right)\right)
$$

where $\psi_{m}$ acts on $T_{\tau}$ by $z \rightarrow m \cdot z$. Now we see (12.21) exhibiting $\tau_{q}^{\prime}(M)$ as a meromorphic section $\mathscr{S}_{\tau}^{\prime}$ of $\left(L_{\tau}^{\prime}\right)^{\varepsilon}$ where $\varepsilon=0$ if the $S^{1}$ action lifts to the spin bundle, and otherwise $\varepsilon= \pm 1$. (Note that $\varepsilon$ is computed at any component of the fixed point set by $\varepsilon=\left(\sum_{i} m_{i} \cdot \operatorname{dim} E_{m_{i}}\right)_{\bmod 2}$ and Lemma 9.3 guarantees that this computation is independent of the choice of component.) Thus we have verified Lemma 12.2.

Actually, (12.21) does more for us. We can conclude, as in $\S \S 7$ and 8 , that $\mathscr{S}_{\tau}^{\prime}(M)$ has no poles on the circle $\left|\lambda=e^{2 \pi i z_{\tau}}\right|=1$ since the left-hand side of $(12.21)$ is, a priori, a power series in $q$ with coefficients that are finite Laurent series in $\lambda^{1 / 2}$.

The possible poles of $\mathscr{S}_{\tau}^{\prime}(M)$ can occur only at points on $T_{\tau}$ where $\psi_{m} \chi_{\tau}$ has poles and thus only on circles in $T_{\tau}$ of the form

$$
a=\alpha^{s} \quad \text { with } \quad \alpha=e^{2 \pi i \tau / k} .
$$

Furthermore, a transfer formula that is analogous to (7.5) and (7.12) will insure the regularity of $\mathscr{S}_{\tau}^{\prime}$ on these circles by identifying the translation of $\mathscr{S}_{\tau}^{\prime}$ by $\alpha^{s}$ with the Chern character of the index of an appropriate elliptic operator on the submanifold $M_{k} \subseteq M$ fixed by $\mathbf{Z}_{k} \subset S^{1}$. Once the transfer formula is exhibited, the argument goes just as for $\tau_{q}(M)$. With this clear, we complete the rigidity proof by giving the transfer formula.

Once again, the formulae for $k$ even and for $k$ odd are slightly different, so we will treat these cases separately. When $k$ is odd, (7.4) describes the decomposition of $T$ along $M_{k}$, and since $T$ is spin, (7.4) insures that $T M_{k}$ is $\operatorname{Spin}_{\mathbf{C}}$ with complex line bundle $L=\bigotimes_{r=1}^{(k-1) / 2} \operatorname{det} T_{r} \rightarrow M_{k}$. For each $r \in\{1, \ldots,(k-1) / 2)\}$ and integer $s$ relatively prime to $k$, we set

$$
\omega(r)=(s \cdot r)_{\bmod k} \in\{1, \ldots, k-1\} .
$$

Then the $k$ odd transfer formula is

$$
t_{\alpha^{s}} \mathscr{S}_{\tau}^{\prime}=\alpha^{b} \cdot \tau_{q}^{\prime}\left(M_{k L}^{\prime} ;\left.\bigotimes_{r=1}^{(k-1) / 2} \hat{\chi}_{\tau}\left(T_{r}\right)\right|_{z=(\omega(r) / k) \cdot \tau}\right),
$$

where $b=\frac{1}{2} \sum_{r=1}^{(h-1) / 2} \omega(r) \cdot \operatorname{dim}_{\mathrm{C}} T_{r}$ and where the oriented manifold $M_{k}^{\prime}$ is defined to be the manifold $M_{k}$ but oriented as in (8.13).

The proof of (12.24) goes just like the proof of the $k$ odd case of (8.13). Indeed, the crucial identity for (8.13) was the periodicity relation $\varphi(\lambda \varphi)=$ $-\varphi(\lambda)$ which has its analogue for $\chi_{\tau}$ in (12.10). 
When $k$ is even, the proof is more complicated because of how $\left.T\right|_{M_{k}}$ decomposes in (7.11). Here $T M_{k} \oplus T_{k / 2}$ is $\operatorname{Spin}_{\mathrm{C}}$ using the line bundle $L=$ $\bigotimes_{r=1}^{k / 2-1}$ det $T_{r} \rightarrow M_{k}$, but $T M_{k}$ itself may not be $\operatorname{Spin}_{\mathrm{C}}$ for any line bundle over $M_{k}$. Thus, in the $k$ even case, we must replace the possibly nonexistent Spin $_{C}$ Dirac operator $d_{S} \otimes \Delta\left(T M_{k}^{\prime} ; L\right)^{-1}$ that appears in (12.16) and (12.17) by

$$
d_{S} \otimes \Delta\left(T M_{k}^{\prime} \oplus T_{k / 2} ; L\right)^{-1}
$$

With this replacement implicitly understood, the $k$ even transfer formula is

$$
t_{\alpha^{s}} \mathscr{S}_{\tau}^{\prime}=\alpha^{b} \cdot \tau_{q}^{\prime}\left(M_{k L}^{\prime} ;\left.\bigotimes_{r=1}^{k / 2-1} \hat{\chi}_{\tau}\left(T_{r}\right)\right|_{z=(\omega(r) / k) \cdot \tau} \otimes R_{q}^{\prime \prime}\left(T_{k / 2}\right)\right)
$$

where $b=\frac{1}{2} \sum_{r=1}^{k / 2-1} \omega(r) \operatorname{dim}_{\mathbf{C}} T_{r}+\frac{1}{4} \omega(k / 2) \operatorname{dim}_{\mathbf{R}} T_{k / 2}$ and where

$$
R_{q}^{\prime \prime}\left(T_{k / 2}\right)=\Delta\left(T_{k / 2} \otimes_{\mathbf{R}} \mathrm{C}\right) \bigotimes_{n=1}^{\infty} \Lambda_{q^{n}} T_{k / 2} \bigotimes_{m=1}^{\infty} S_{q^{m-1 / 2}} T_{k / 2}
$$

Also, the oriented manifold $M_{k}^{\prime}$ in (12.26) is the manifold $M_{k}$, but oriented as in (8.13).

The proof of (12.26) goes like the proof of (8.13), and we leave it to the reader.

\section{ON THE MODULAR PROPERTY OF $\tau_{q}(M)$ AND $\tau_{q}^{\prime}(M)$}

At this time there are already several fine accounts explaining the modular nature of $\tau_{q}(M)$, see [Br, C-C, $\left.\mathrm{La}^{*}, \mathrm{M}, \mathrm{S}, \mathrm{Z}\right]$. Nevertheless, it might be appropriate to explain this phenomenon in our context at least in the isolated fixed point case.

Let us then assume that $M$ is spin so that $\tau_{q}(M)$ is constant on $T_{q^{2}}$.

From the fixed point formula we know that this constant can be computed by summing the values of the fixed point contributions $\mu_{p}(a)$ at any point $a \in T_{q^{2}}$. Of course if $\mu_{p}$ has a pole at $a, \mu_{p}(a)$ must be taken to be the constant term in its Laurent series at $a$. To apply this procedure at $\lambda=1$ we introduce $e^{z}=\lambda$ so that $z$ is a local parameter near $\lambda=1$ and recall here that $\varphi$ has a pole of order 1 at $\lambda=1$ and is odd in the sense that $\varphi\left(\lambda^{-1}\right)=-\varphi(\lambda)$. So it follows that

$$
\varphi\left(e^{z}\right)=\frac{K}{z}\left\{1+a_{2} z^{2}+a_{4} z^{4}+\cdots\right\}
$$

near $z=0$.

Now recall that

$$
\mu_{p}\left(e^{z}\right)=\bigotimes_{1}^{d} \psi_{m^{i}} \varphi\left(e^{z}\right)=\bigotimes_{1}^{d} \varphi\left(e^{m_{i} z}\right)
$$


so after expanding to obtain the constant term, one finds that

$$
\mu_{p}(1)=K^{d} \cdot \sum_{\alpha} \frac{L_{\alpha}(m)}{\prod m_{i}} \cdot a^{\alpha},
$$

where $a^{\alpha}$ is a monomial of weight $\operatorname{dim} M=2 d$ in the $a_{j}$, those being weighted by $2 j$, and $L_{\alpha}(\cdot)$ is a universal symmetric polynomial in $m_{j}^{2}$ of weight $2 d$. Now the sums

$$
L_{\alpha}(M)=\sum_{p} \frac{L_{\alpha}(m)}{m_{1} \cdots m_{d}}
$$

are known to yield the characteristic numbers, associated by the polynomial $L_{\alpha}(\cdot)$ to $M$. (See [A-B ${ }^{*}$ ] or [B].) Hence this procedure establishes $\tau_{q}(M) \cdot K^{-d}$ as a universal polynomial in the $a_{j}$ with integral coefficients that are Pontrjagin numbers of $M$ :

$$
\tau_{q}(M) \cdot K^{-d}=\sum L_{\alpha}(M) a^{\alpha}
$$

Here $K$ is a normalizing factor which we already computed in $\S 5$. It is

$$
K=2 \cdot \frac{\Pi\left(1+q^{n}\right)^{2}}{\Pi\left(1-q^{n}\right)^{2}}
$$

For example, when $\operatorname{dim} M=4$, the constant term of $\mu_{p}$ at 1 is clearly $\left\{\left(m_{1}^{2}+m_{2}^{2}\right) / m_{1} m_{2}\right\} \cdot a_{2}$. Hence in that case

$$
\tau_{q}(M) \cdot K^{-2}=p_{1}(M) \cdot a_{2} .
$$

In dimension 8 one calculates similarly to find

$$
\begin{aligned}
\tau_{q}(M) \cdot K^{-4} & =p_{2} \cdot a_{2}^{2}+\left(p_{1}^{2}-2 p_{2}\right) a_{4} \\
& =p_{1}^{2} a_{4}+p_{2}\left(a_{2}^{2}-2 a_{4}\right)
\end{aligned}
$$

etc.

Now the formula (13.5) immediately establishes $\tau_{q}(M) \cdot K^{-d}$ as a modular form for the subgroup $\Gamma_{0}(2)$, of $S L(2, \mathbf{Z})$, of matrices with even lower left entry.

Indeed, the function $\varphi_{*}=\varphi / K$ is canonical, up to a scalar multiple, on tori with a singled out point of order 2 . Therefore, the coefficients of its Laurent expansion near its pole, in short our $a_{j}$, are modular forms for $\Gamma_{0}(2)$, whose weight can be read off to be $2 j$ by simply keeping track of how they transform under $z \rightarrow \lambda z$.

Actually, more is true. When $p_{1}(M)=0$ then $\tau_{q}(M) \cdot K^{-d}$ is actually modular for all of $S L(2, \mathbf{Z})$. To see this, note that the Weierstrass $\mathscr{P}$-function is the canonical elliptic function on a torus $T$ which near $1 \in T$ has the behavior

$$
\mathscr{P}(z)=\frac{1}{z^{2}}+b_{2} z^{2}+b_{4} z^{6}+\cdots
$$


Because $\mathscr{P}(z)$ is so canonical, we see that the $b_{k}$ are automorphic forms of degree $2(k+2)$ for all of $S L(2, \mathbf{Z})$. Thus, $b_{2}$ is of degree 4 and $b_{4}$ of degree 6.

But note now that $\varphi_{*}$, when squared, has the polar behavior of the $\mathscr{P}$ function when constructed with our local parameter $z$ of the torus $T_{q}$. Hence

$$
\varphi_{*}^{2}=\mathscr{P}-e
$$

where $e=\mathscr{P}(-1)$. Equating powers of $z$ we get equalities

$$
\begin{aligned}
2 a_{2} & =-e, \\
2 a_{4}+a_{2}^{2} & =b_{4}, \\
2\left(a_{2 k}+\sum_{r+s=k} a_{2 r} \cdot a_{2 s}\right)+a_{k / 2}^{2} & =b_{2 k}, \quad k>2 .
\end{aligned}
$$

From these we observe inductively that modulo the ideal generated by $e=2 a_{2}$, the higher $a$ 's are determined by the $b$ 's.

$$
2 a_{4} \equiv b_{4}, \quad 2 a_{6} \equiv b_{6}, \ldots
$$

But from this it is not difficult to argue that if $p_{1}(M)=0$ then $K^{-\operatorname{dim} M / 2}$. $\tau_{q}(M)$ is a linear combination of the $b_{2 j}$ and hence a modular form for all of $S L(2, \mathbf{Z})$.

When $S^{1}$ acts with isolated fixed points, the effect of $S L(2, \mathbf{Z})$ on $\tau_{q}^{\prime}(M)$ can be analyzed by similar methods. For this, we go to our covering space, the upper half plane $H$, and we label complex tori as $T_{\tau}, \tau \in H$. Then the square of our section $\chi_{\tau}$ of $L_{\tau}$ in (12.10) is a meromorphic function on $T_{\tau}$ with a zero of order 2 at $(1+\tau) / 2$ and a pole of order 2 at 0 with residue $K^{\prime 2}$ where

$$
K^{\prime}=\prod_{n=1}^{\infty} \frac{\left(1+q^{n-1 / 2}\right)^{2}}{\left(1-q^{n}\right)^{2}}
$$

Thus, we can write $\chi_{\tau *}=\chi_{\tau} / K^{\prime}$ in terms of the Weierstrass $\mathscr{P}$-function as

$$
\chi_{\tau *}^{2}=\mathscr{P}-e^{\prime}
$$

where $e^{\prime}=\mathscr{P}\left(z_{\tau}=(1+\tau) / 2\right)$. Now a repeat of our analysis for $\varphi_{*}$ shows that $\left(K^{\prime}\right)^{-\operatorname{dim} M / 2} \cdot \tau_{q}^{\prime}(M)$ is also fully modular under $S L(2, \mathbf{Z})$ when $p_{1}(M)=0$.

The identities in (13.10) and (13.13) are important for another reason. They indicate a proof of a general relationship (noted by Witten) between $\tau_{q}(M)$ and $\tau_{q}^{\prime}(M)$. To state the relationship, we write $q=e^{2 \pi i \tau}$ and $q^{*}=e^{-2 \pi i /(\tau+1)}$. Then recall that the generators of $S L(2, \mathbf{Z})$ on $\mathbf{R}^{2} / \mathbf{Z} \times \mathbf{Z}, e_{1}$ and $e_{2}$ of (12.6), act so that $e_{1}^{*} e_{2}^{*} L_{-1 /(\tau+1)}=L_{\tau}^{\prime}$ as an identification of flat line bundles over $T_{\tau}$.

With this in mind, the relationship between $\tau_{q}$ and $\tau_{q}^{\prime}$ is

$$
e_{1}^{*} e_{2}^{*}\left(K^{-\operatorname{dim} M / 2} \cdot \tau_{q^{*}}(M)\right)=K^{\prime-\operatorname{dim} M / 2} \cdot \tau_{q}^{\prime}(M) .
$$


Note that (13.14) verifies the rigidity of $\tau_{q}^{\prime}(M)$ given the rigidity of $\tau_{q}(M)$.

Now a proof of (13.14) will take us too far afield to present, except for the case when $S^{1}$ acts on $M$ with isolated fixed points. In this case, both sides of (13.14) are expressed, using the fixed point formulae, in terms of $\varphi_{\tau}$ or $\chi_{\tau}$, and (13.10) and (13.13) suggest that $S L(2, \mathbf{Z})$ relates these two objects. Indeed, one has

$$
e_{1}^{*} e_{2}^{*}\left(K^{-1} \varphi_{-1 /(\tau+1)}\right)=K^{\prime-1} \chi_{\tau},
$$

as can be verified by comparing the zeros and poles of both sides. Furthermore, the action of $S L(2, \mathbf{Z})$ on $\mathbf{R}^{2} / \mathbf{Z} \times \mathbf{Z}$ commutes with multiplication by integers, so (13.15) remains true with $\psi_{m} \varphi$ and $\psi_{m} \chi$ replacing $\varphi$ and $\chi$. With the fixed point formulae, these observations immediately yield (13.14) in the isolated fixed point case.

\section{REMARKS ON THE ALMOST COMPLEX CASE}

We close with a few indications of how the preceding discussion extends to $S^{1}$ actions on almost complex manifolds. Essentially one finds new rigidity phenomena once $c_{1}(M)$ is divisible by $N$.

Recall that on such a manifold the cocomplexified tangent bundle splits,

$$
T^{*} \otimes \mathrm{C}=T^{\prime} \oplus T^{\prime \prime},
$$

into a "holomorphic" and "antiholomorphic" part and that the deRham $d$ splits correspondingly into

$$
d=\partial^{\prime}+\partial^{\prime \prime}
$$

On such manifolds

$$
\partial^{\prime \prime}: \Lambda^{0, \text { even }} \rightarrow \Lambda^{0, \text { odd }}
$$

can be taken to be the dominant operator in the sense that $d+d^{*}, d_{S}$, and $\not \partial$, where defined, are now appropriate twists of $\bar{\partial}$. Namely,

$$
\not \partial=\partial^{\prime \prime} \otimes\left(\operatorname{det}\left(T^{\prime}\right)\right)^{1 / 2}, \quad d_{S}=\partial^{\prime \prime} \otimes \Lambda_{1}\left(T^{\prime}\right)
$$

and

$$
d+d^{*}=\partial^{\prime \prime} \otimes \Lambda_{-1}\left(T^{\prime}\right)
$$

The factorization of $d_{S}$ leads one to suspect that the operators

$$
d_{S}^{(\alpha)}=\bar{\partial} \otimes \Lambda_{\alpha} T^{\prime}
$$

with $\alpha$ a root of 1 should now be amenable to a similar treatment as $d_{S}$ was. This is indeed the case. Namely, consider the $\theta$-function

$$
\theta_{\alpha}(\lambda)=(1+\alpha \lambda) \bigotimes_{n=1}^{\infty}\left(1+\alpha q^{n} \lambda\right)\left(1+\alpha^{-1} q^{n} \lambda^{-1}\right)
$$


We then have

$$
\theta_{\alpha}(q \lambda)=\alpha^{-1} \lambda^{-1} \theta(\lambda)
$$

so that the expression

$$
\varphi_{\alpha}(\lambda) \equiv \theta_{\alpha}(\lambda) / \theta_{-1}(\lambda)
$$

transforms by

$$
\varphi_{\alpha}(q \lambda)=-\alpha^{-1} \varphi_{\alpha}(\lambda) .
$$

Hence if $-\alpha$ is an $N$ th root of 1 then $\varphi_{\alpha}(\lambda)$ naturally descends to a rational function on $T_{q^{N}}$. So interpreted, $\varphi^{N}$ becomes canonical, up to a constant, on tori with a singled out $N$ th root, $\omega$, of 1 . It is characterized by the fact that it has poles only at the powers of $\omega$ and that translation by $\omega$ multiplies the function by $-\alpha^{-1}$.

These considerations now lead us to define the following auxiliary $q$-bundle $R_{q}^{\alpha}$ on almost complex manifolds

$$
R_{q}^{\alpha}=\Lambda_{\alpha} T^{\prime} \bigotimes_{n=1}^{\infty}\left(\Lambda_{\alpha q^{n}} T^{\prime} \otimes \Lambda_{\alpha^{-1} q^{n}} T^{\prime *} \otimes S_{q^{n}} T^{\prime} \otimes S_{q^{n}} T^{\prime *}\right)
$$

The rigidity phenomenon, also essentially predicted by Witten, in this context is now given by

Theorem. On an almost complex manifold $M$ with $c_{1} \equiv 0 \bmod N$, the operators $\partial^{\prime \prime} \otimes R_{q}^{\alpha}$ are rigid provided $(-\alpha)^{N}=1$.

Note that for $\alpha=1, N=2$, we recover the rigidity of $\tau_{q}(M)$ in this almost complex case.

There is also an $R_{q}^{\prime}$ rigid relative to the operator $\partial^{\prime \prime} \otimes\left(\operatorname{det} T^{\prime}\right)^{1 / N}$ defined on almost complex manifolds with $c_{1} \equiv 0 \bmod N$. It generalizes $R_{q}^{\prime}$ of $(2.11)$ and is given by

$$
R_{q}^{\prime N}=R_{q}^{N}
$$

but with $(-\alpha)^{N}=q$.

We will discuss the details, which run entirely parallel to our discussion so far, at some other time.

\section{REFERENCES}

[A] L. V. Ahlfors, Complex analysis, 3rd ed., McGraw-Hill, New York, 1979.

[A-H] M. F. Atiyah and F. Hirzebruch, Spin manifolds and group actions, Essays in Topology and Related Subjects, Springer-Verlag, Berlin, 1970, pp. 18-28.

[A-B] M. F. Atiyah and R. Bott, The Lefschetz fixed point theorem for elliptic complexes I and II, Ann. of Math. 86 (1967), 374 and 88 (1968), 451.

[A-B*] _ The moment map and equivariant cohomology, Topology 23 (1984), 1.

[A-S] M. F. Atiyah and G. Segal, The index of elliptic operators II, Ann. of Math. 87 (1968), 531. 
[B] R. Bott, On the fixed point formula and the rigidity theorems of Witten, Lectures at Cargèse, 1987 (to appear).

[B-S] R. Bott and H. Samelson, Applications of the theory of Morse to symmetric spaces, Amer. J. Math. 80 (1958), 964.

[Br] J.-L. Brylinski, Remark on Witten's modular forms, 1987, preprint.

[C-C] D. V. Chudnovsky and G. V. Chudnovsky, Elliptic modular functions and elliptic genera, Topology (to appear).

[E] A. L. Edmonds, Orientability of fixed point sets, Proc. Amer. Math. Soc. 82 (1981), 120.

$[\mathrm{H}]$ F. Hirzebruch, Elliptic genera of level $N$ for complex manifolds, 1988, preprint.

[La] P. S. Landweber, Elliptic genera: An introductory overview, Elliptic Curves and Modular Forms in Algebraic Topology (P. S. Landweber, ed.), Lecture Notes in Math., no. 1326, SpringerVerlag, Berlin and New York, 1988, pp. 1-10.

$\left[\mathrm{La}^{*}\right]$ _ Elliptic cohomology and modular forms, Elliptic Curves and Modular Forms in Algebraic Topology (P. S. Landweber, ed.), Lecture Notes in Math., no. 1326, Springer-Verlag, Berlin and New York, 1988, pp. 55-68.

[L-S] P. S. Landweber and R. E. Stong, Circle actions on spin manifolds and characteristic numbers, Topology 37 (1988), 45.

[M] H. Miller, The elliptic character and the Witten genus, 1988, preprint.

[O] S. Ochanine, Genres elliptiques équivariants, Elliptic Curves and Modular Forms in Algebraic Topology (P. S. Landweber, ed.), Lecture Notes in Math., no. 1326, Springer-Verlag, Berlin and New York, 1988, pp. 107-122. Also in unpublished notes of a 1985 lecture.

[S] G. Segal, Elliptic cohomology, Séminaire Bourbaki, 40e année, 1987-88, no. 695 (to appear).

[T] C. H. Taubes, $S^{1}$-actions and elliptic genera, 1987, preprint.

[W] E. Witten, Elliptic genera and quantum field theory, Comm. Math. Phys. 109 (1987), 525 and The index of the Dirac operator on loop space, Elliptic Curves and Modular Forms in Algebraic Topology (P. S. Landweber, ed.), Lecture Notes in Math., no. 1326, Springer-Verlag, Berlin and New York, 1988, pp. 161-181.

[Z] D. Zagier, Note on the Landweber-Stong elliptic genus, Elliptic Curves and Modular Forms in Algebraic Topology (P. S. Landweber, ed.), Lecture Notes in Math., no. 1326, Springer-Verlag, Berlin and New York, 1988, pp. 216-224.

Department of Mathematics, Harvard University, Cambridge, Massachusetts 02138 